\title{
علاقة التوجهات الهدفية بسلوك البحث عن التغذية الراجعة لدى عينة من طلبة الجامعة الهاشمية فِ الأردن

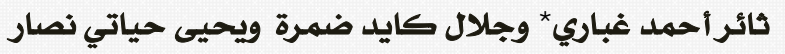 \\ الجامعة الهاشمية، الزرقاء، الأردن
}

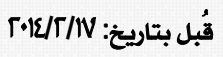

عُدل بتاريخت

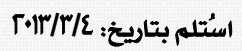

هدفت الدراسة الحالية إلى الكشف عن علاقة التوجهات الهدفية بسلوك البحث عن التغذية الراجعة لدى طلبة الجامعة الهاشمية. تكونت عينة

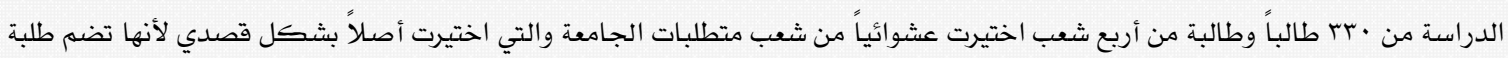
من مختلف الكليات والتخصصات، وقد استجابوا لمقياسي التوجهات الهدفية وسلوك البحث عن التغذية الراجعة. أظهرت النتائج أن التوجهات

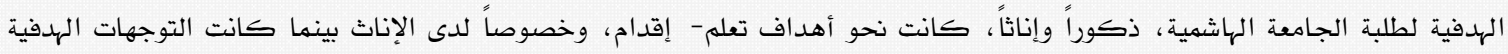

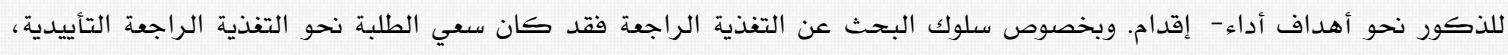

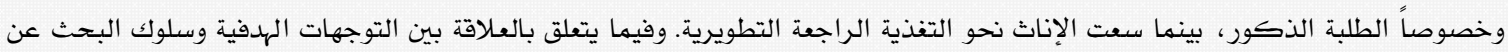

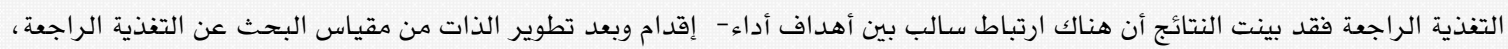

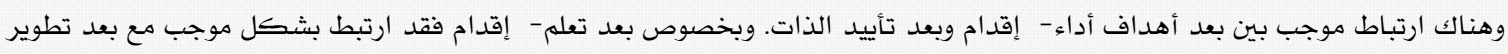

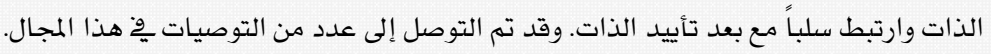
كلمات مفتاحية: التوجهات الهدفية ، سلوك البحث عن التفذية الراجعة ، تطويرالذات، تأييد الذات، الجامعة الهاشمية.

\section{Relationship between $\mathrm{G}$ oal O rientations with Feedback-Seeking Behavior among Sample from the Hashemite University Students}

Thaer A. Ghbari*, Jalal K. Damra \& Yahya H. Nassar The HashemiteUniversity, Zarqa, Jordan

This study aimed at exploring the relationship between goal orientation (GO) and the feedback-seeing behavior (FSB). The sample consisted of 330 students from four sections which were chosen randomly from the university requirements of the Hashemite University. These courses were chosen purposively because they contain students from different faculties and majors. The students completed the GO and the FSB questionnaires. The results showed that the females adopted learning-approach goals, but the males adopted performance-approach goals. Concerning the FSB, students sought self-validation feedback. In respect to gender differences in FSB, males sought self-validation feedback, whereas females sought self-improvement feedback. The results al so showed that there was a negative correlation between performance-approach goals and self-improvement feedback, but there was a positive correlation between performance-approach goals and self-improvement feedback. Learning-approach goals were positively correlated with self-improvement feedback, but correlated negatively with self-validation feedback.

Keyw ords: goal orientation, feedback-seeking behavior, self-improvement feedback, self-validation feedback, HashemiteUniversity.

*thaera@hu.edu.jo 


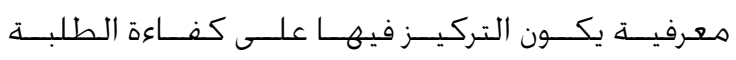

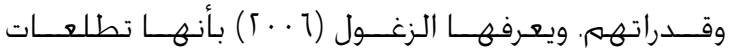

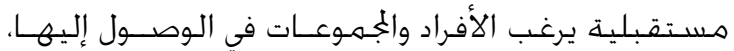

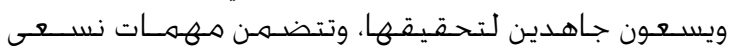

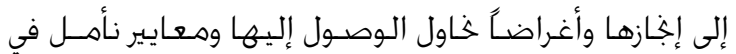

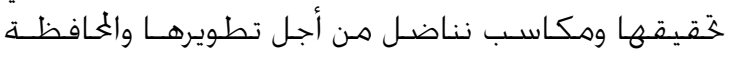

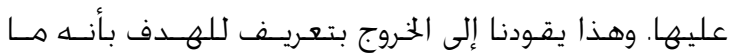

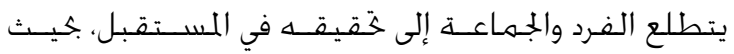

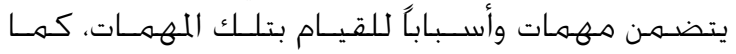
يرافقها معايير للتحقق هـن إنجازها.

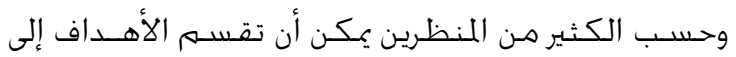

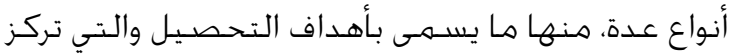

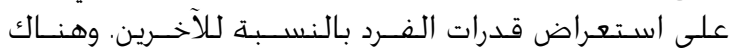

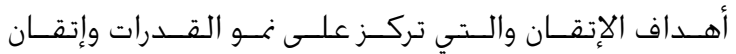
المهارات (Ames \& Archer, 1988; Dweck, 1986; Nicholls, 1984).

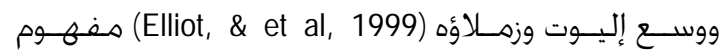

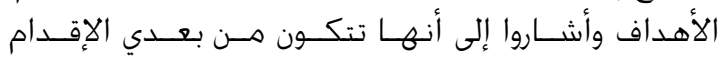

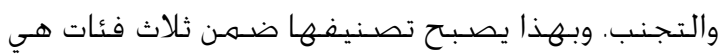

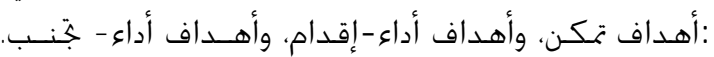

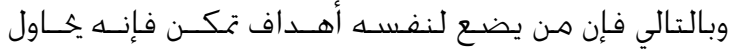

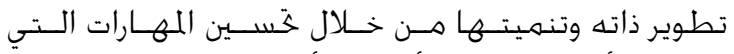

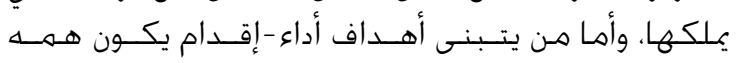

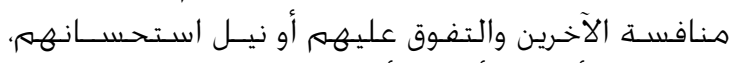

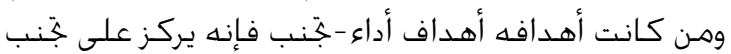

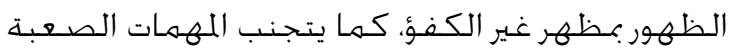

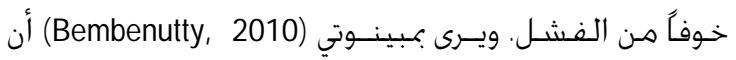

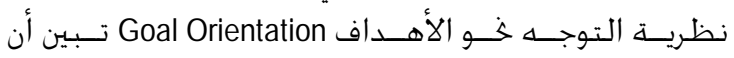

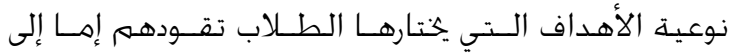

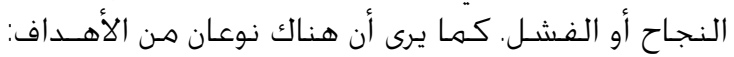
أهداف مـوجهة خهو المهمة، وأهـداف موجهية خهو الأداء.

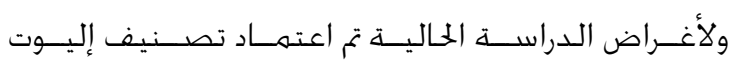

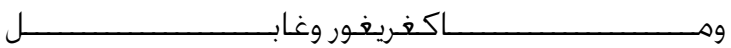
الـــ (Elliot \& McGregor \& Gable, 1999; Elliot, 1999)

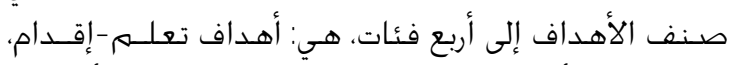

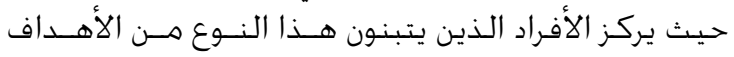

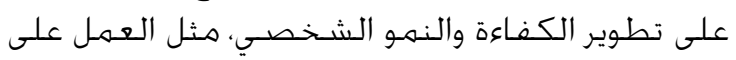

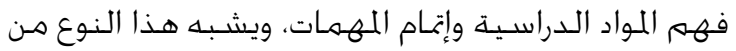

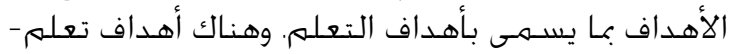

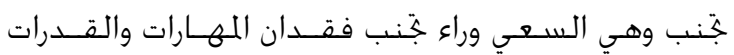

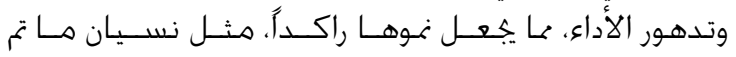

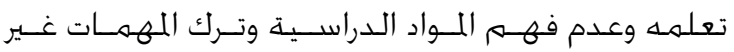

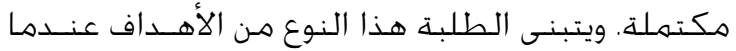

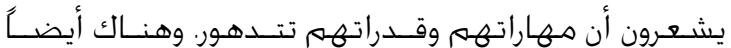

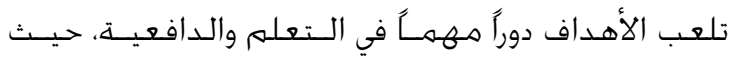

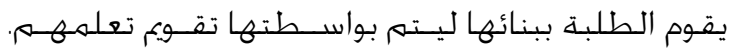

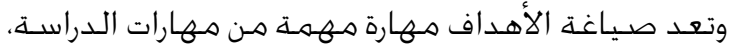

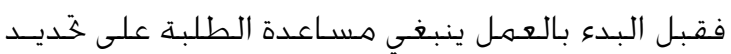

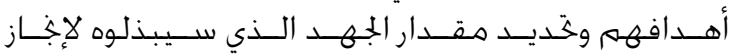

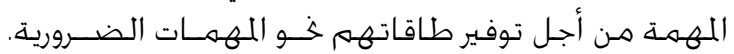

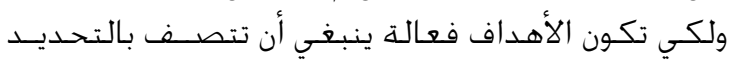

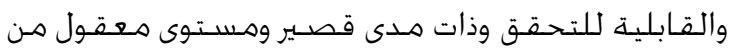

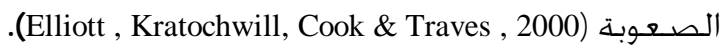

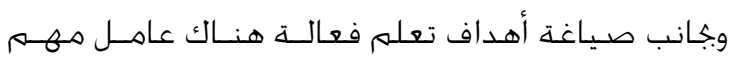

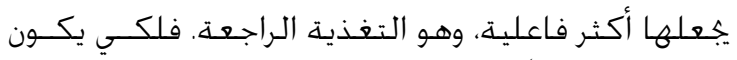

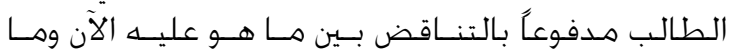

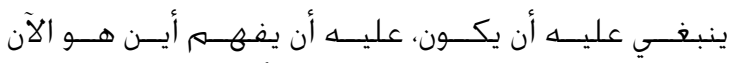

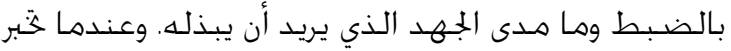

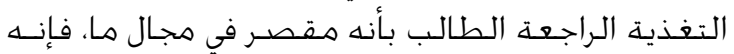
سيبذل قصسارى جهـده وسيـجـرب استـراتيجية أخرى لإجنـاز

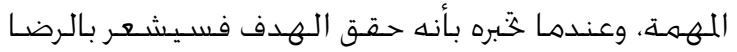

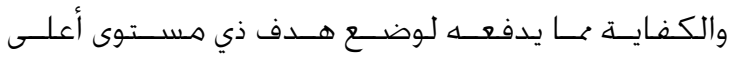

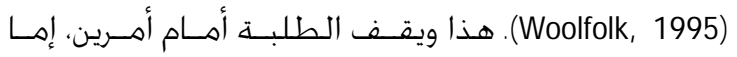

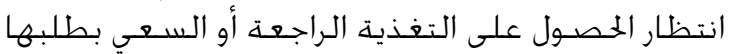

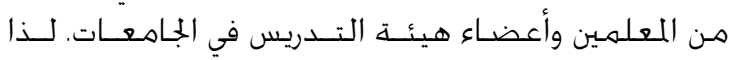

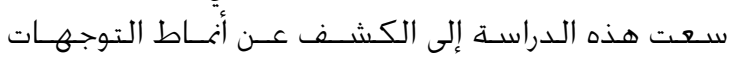

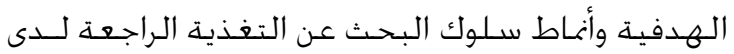

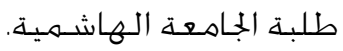

التوجهات الهدفية

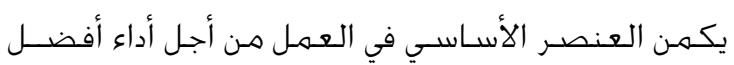

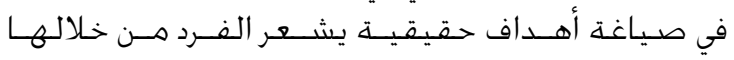

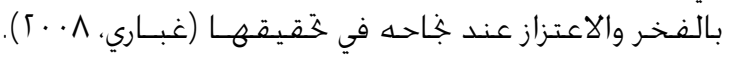

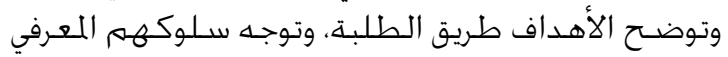

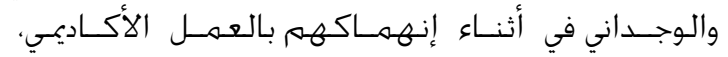

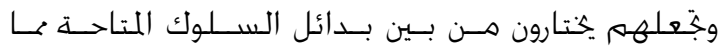

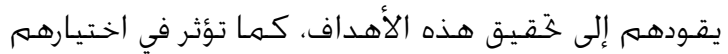

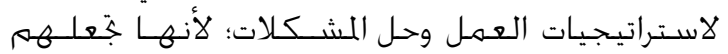

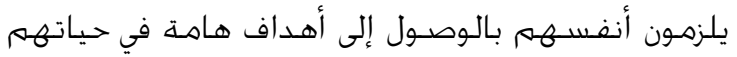

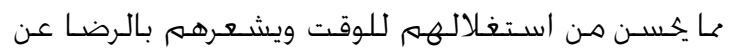

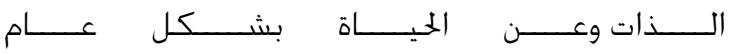
(Ames,1992; Elliot, McGregor \& Gable, 1999; Lock \& .Latham, 1990; Meece, Blumenfeld, \& Hoyle, 1988)

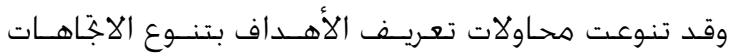

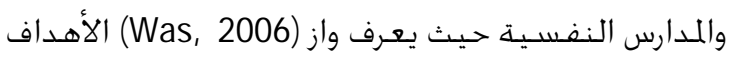

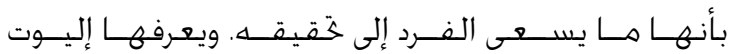

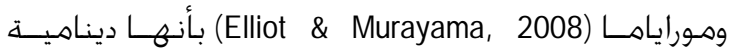


البحـث عـن التغذية الراجعة Feedback Seeking Behavior.

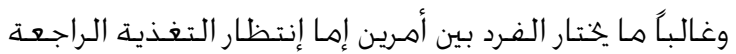

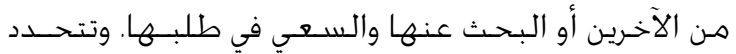

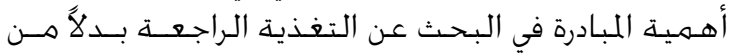

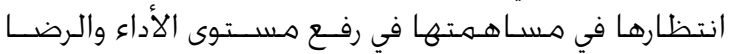

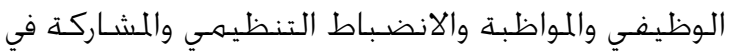

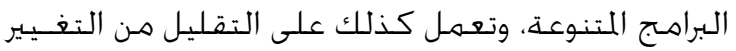

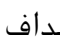
تمهر في الأه

(Ashford \& Cummings, 1983; Ashford, Blatt \& VandeWalle, 2003; Kudisch, Fortunado \& Smith, 2006; (Whitaker, Dahling \& Levy, 2007

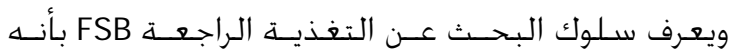

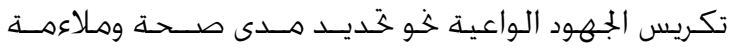

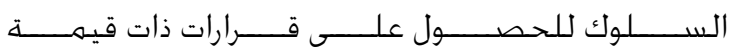

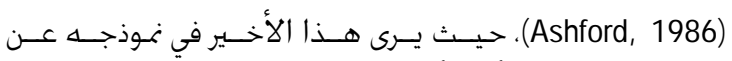

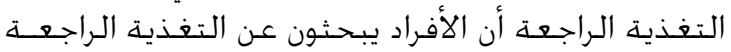

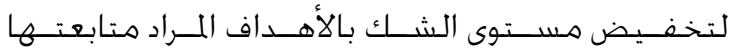

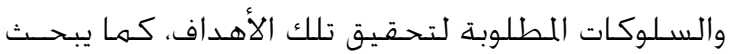

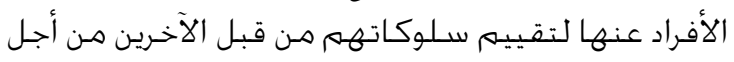

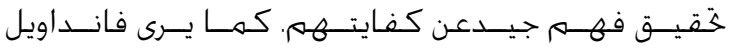
(VandeWalle, 2003)

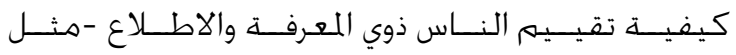

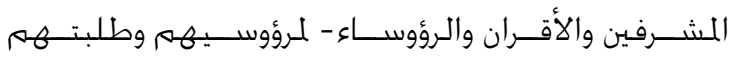
يساعد في تطوير فهمم أعمق لكفـايتهمه. ويعتقــد فانداويل (VandeWalle, 2003) بوجـود ســـة أبعــاد

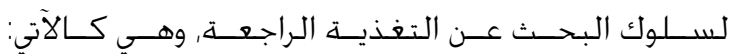

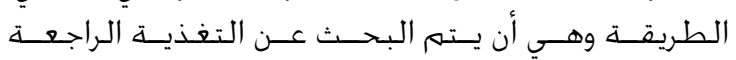

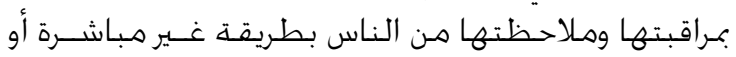

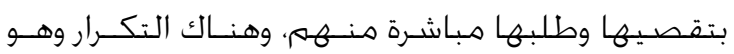

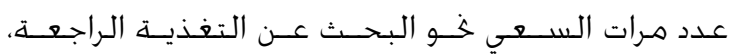

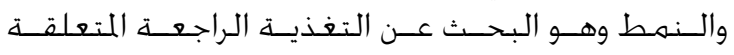

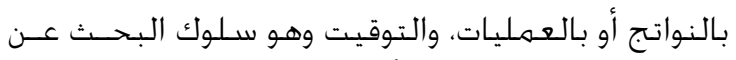

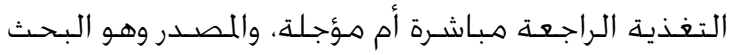

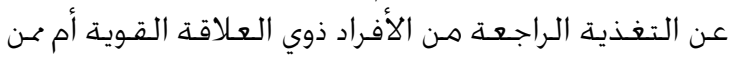

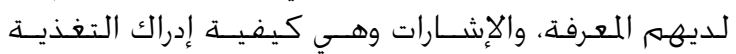
الراجعة إيجابية أم سـلبية.

وريــرى آشــفورد (Ashford, 1986) أن هنــاك اســـراتيجيتـان

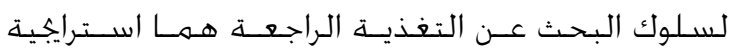

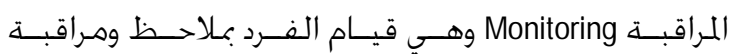

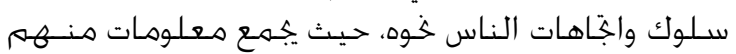

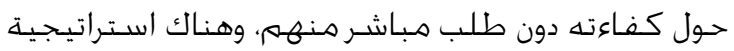

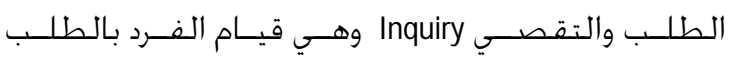

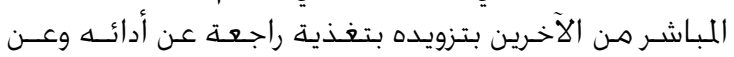

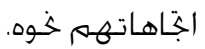

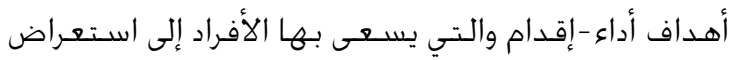

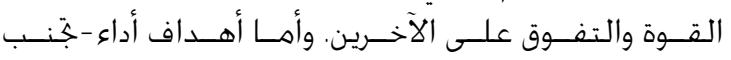

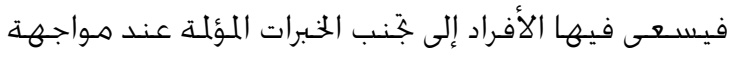

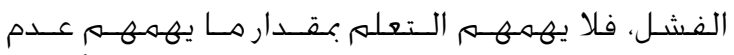

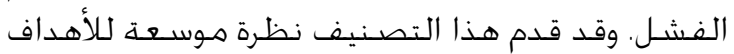

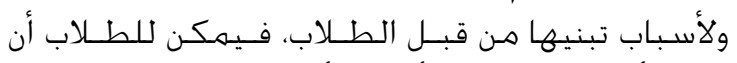

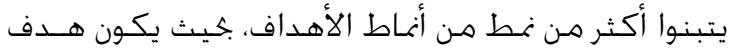

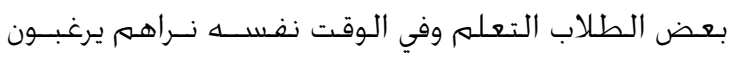

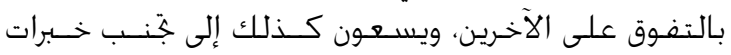

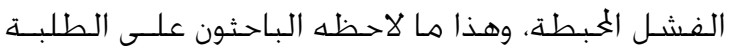

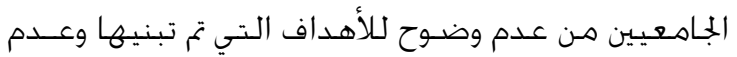

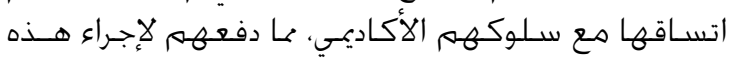

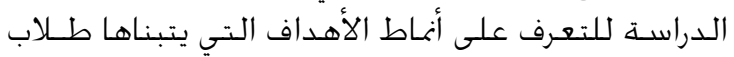

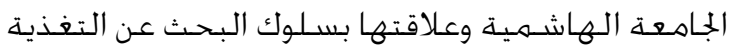

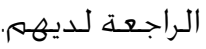

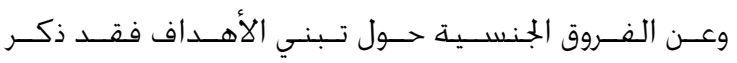

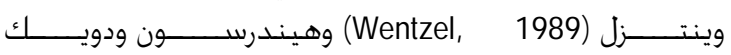
(Hendersson \& Dweck, 1990)

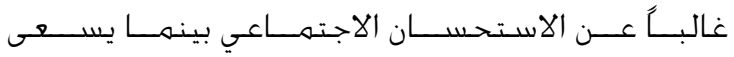

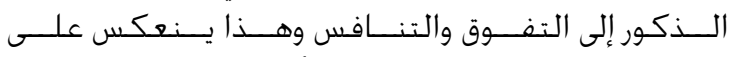

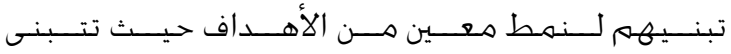

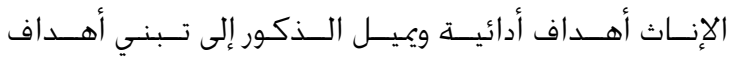

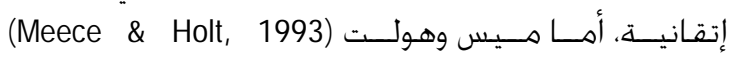

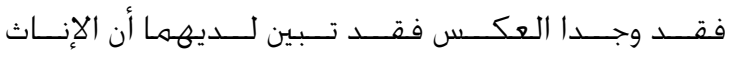

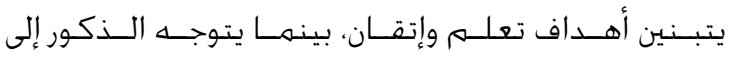
تبنـي أهـداف أدائية.

سـلوك البحث عن التغـية الراجعة

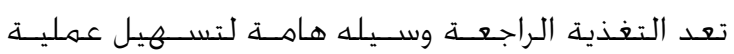

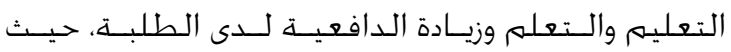

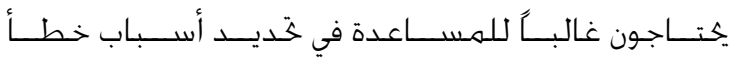

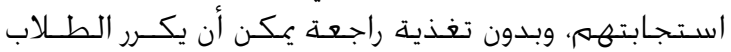

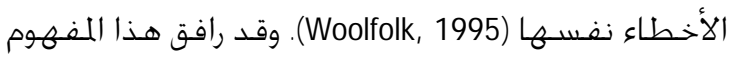

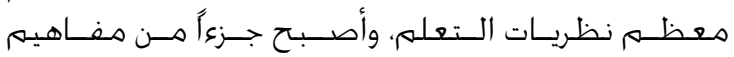

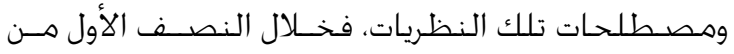

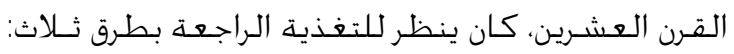

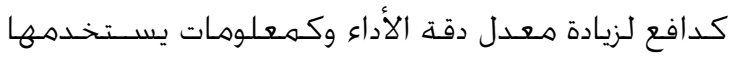

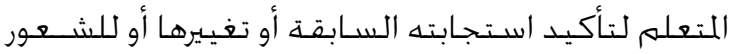

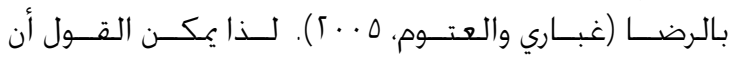

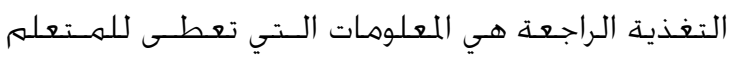

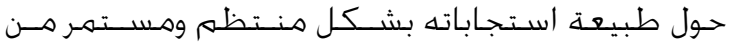

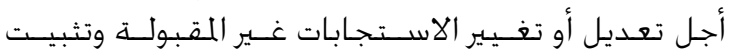

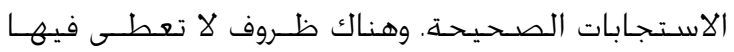

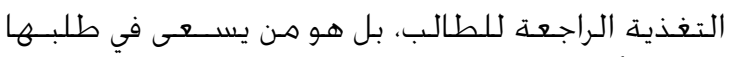

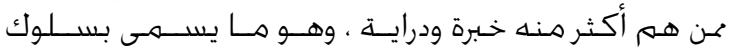


فالأفراد الذين يتبنون أهـداف تعلم يبحثـون عـن التغذيــة

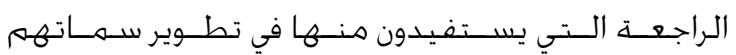

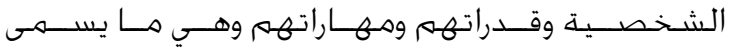

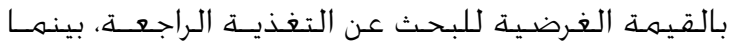

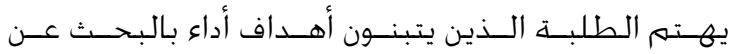

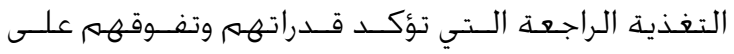

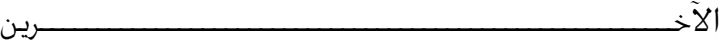
Elliot \& McGregor and Gable, 1999; VandeWalle, ) 2003). وتوصل رين وفيدور (Renn \& Fedor, 2001) إلى ارتباط

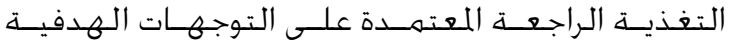

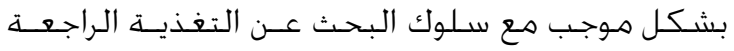
وأداء المهـمات. بل موجن.

الدراسـات السـابقـة

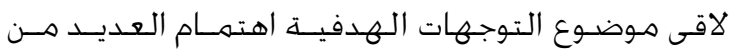

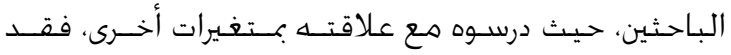

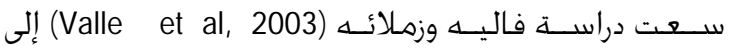

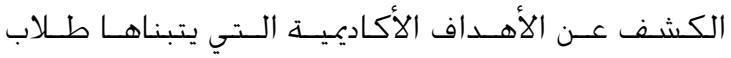

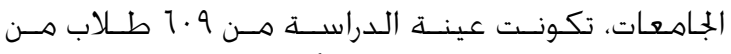

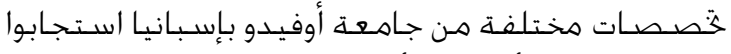

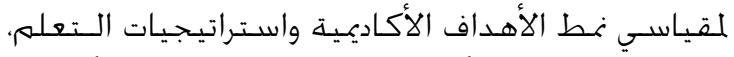

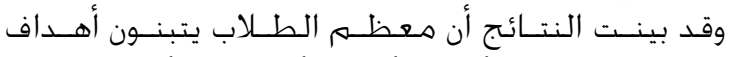

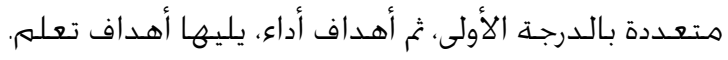

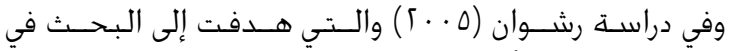

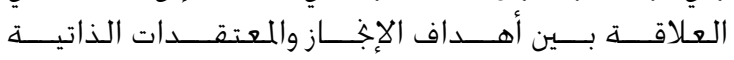

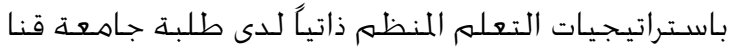

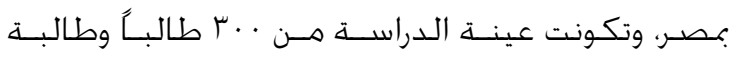

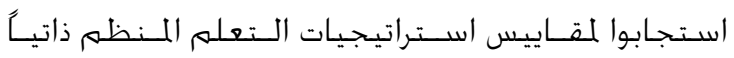

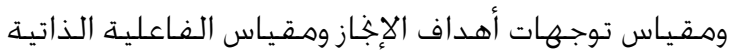

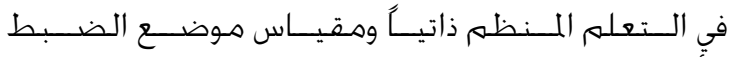

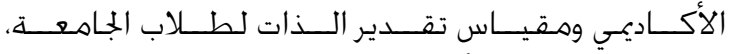

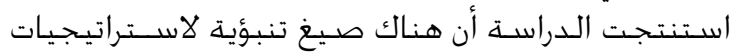

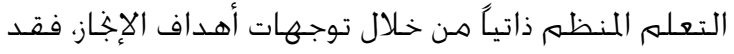

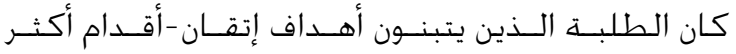

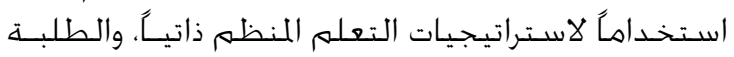

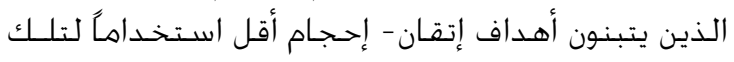
الاسـتراتيجيات.

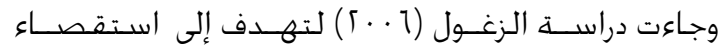

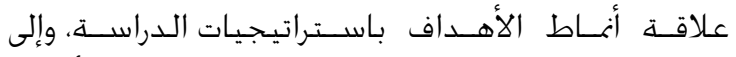

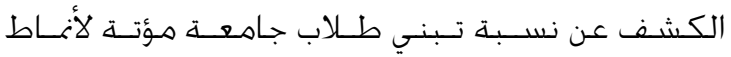

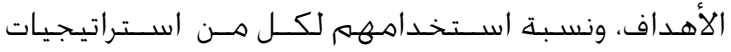

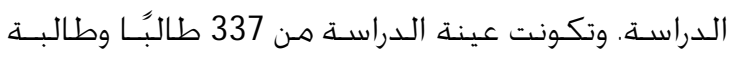

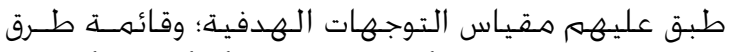

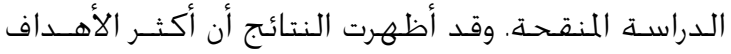

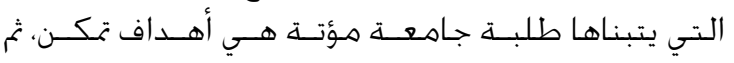

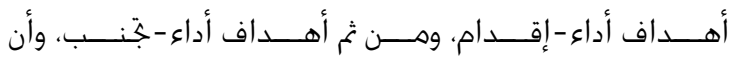

وختدث آشـفورد وكـمنغز (Ashford and Cummings, 1983) عـن

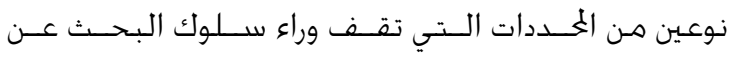

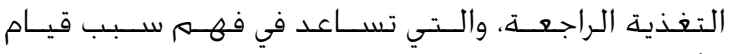

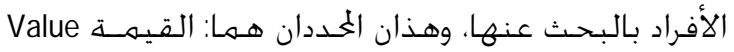

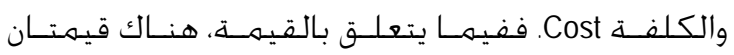

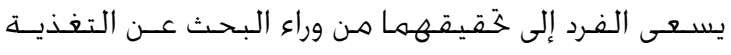

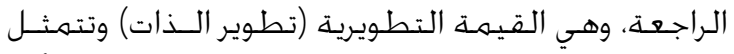

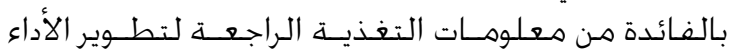

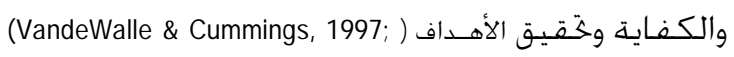
VandeWalle, Ganesan, Challagalla, \& Brown, 2000)

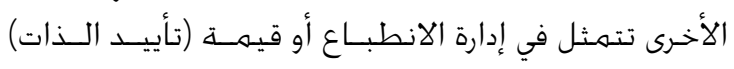

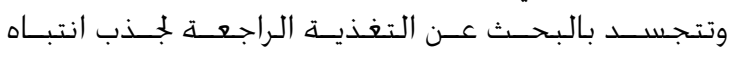

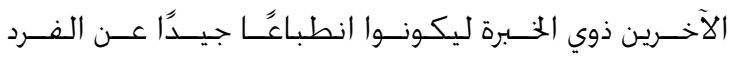
(Ashford, Blatt \& Vande Walle, 2003)

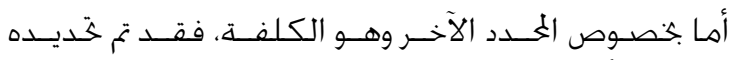

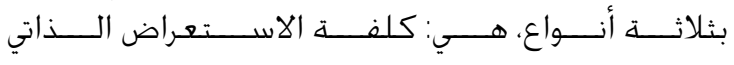
Self- Presenting cost

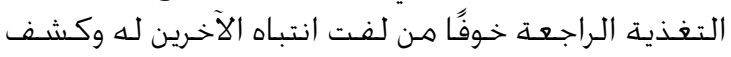

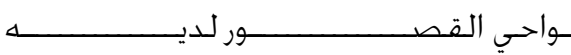

(Ashford, 1986; VandeWalle \& Cummings, 1997) الثاني هو كلفـة الأنا Ego cost وهـي أن يتلقى الفـرد تغذية

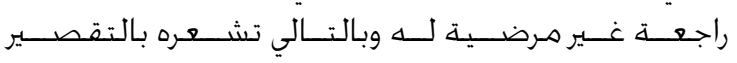

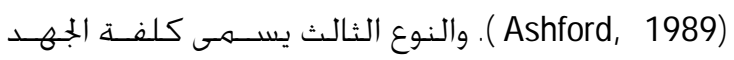

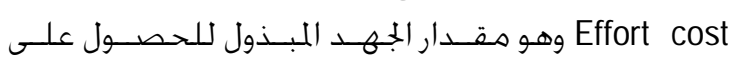
التغذية الراجعة (Ashford, 1986; Ashford \& Cummings, 1983).

علاقـة التوجهات الهـدفية بسـلوك البحث عـن التغذية

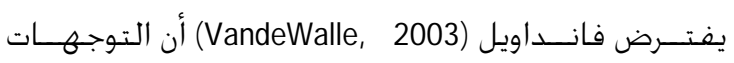

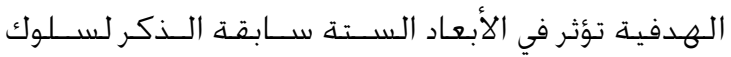

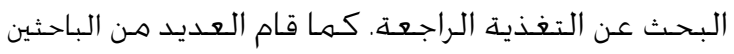
(Janssen \& Prins, 2007; VandeWalle \& Cummings, 1997)

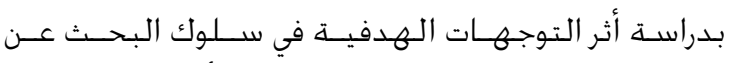

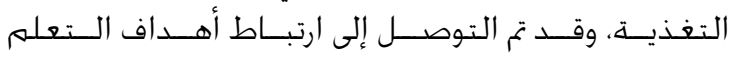

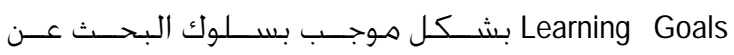

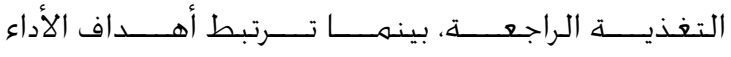

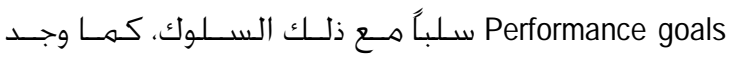

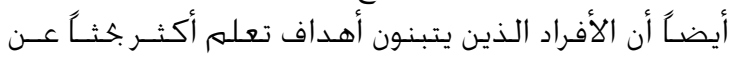

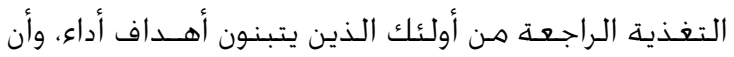

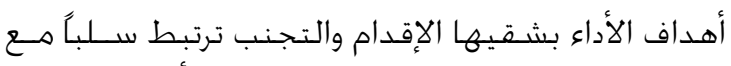

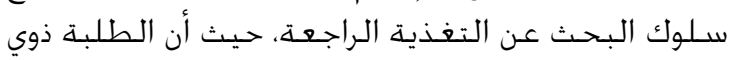

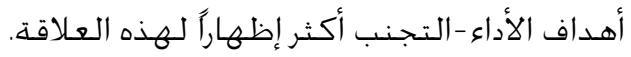

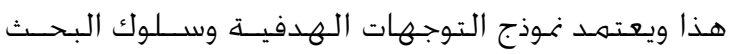

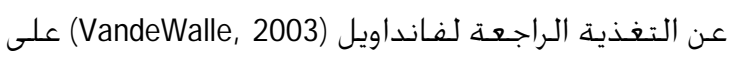

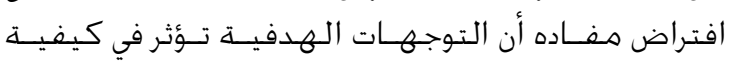

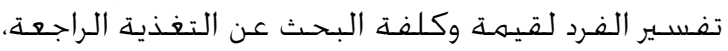




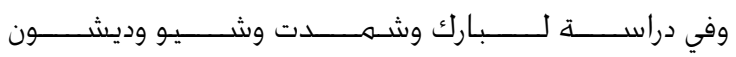
هــــ (Park, Schmidt, Scheu \& DeShon, 2007)

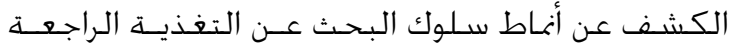

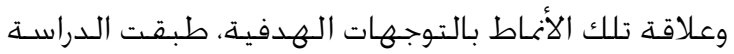

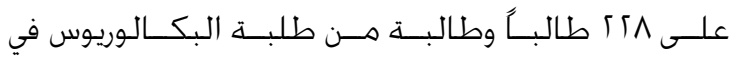

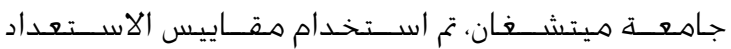

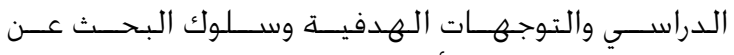

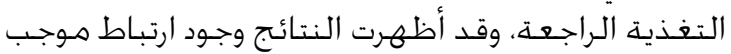

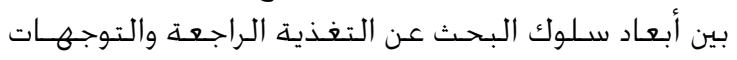

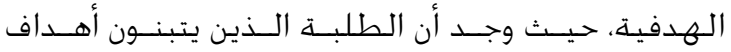

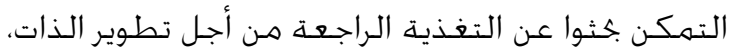

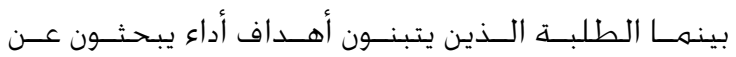
التغذية الراجعة التي تهدف إلى تأييد الذات.

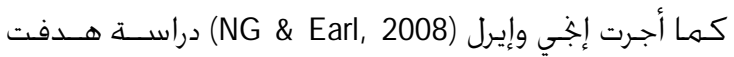

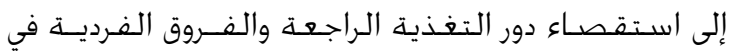

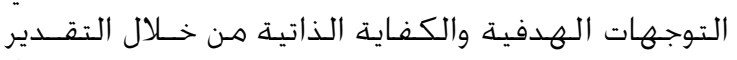

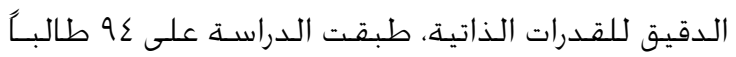

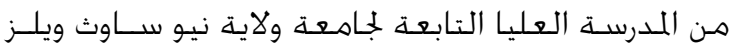

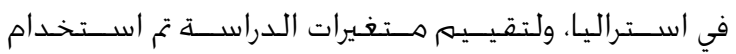

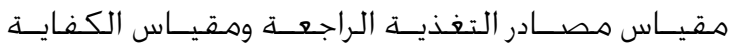

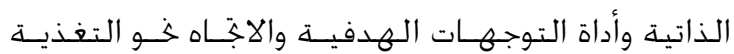

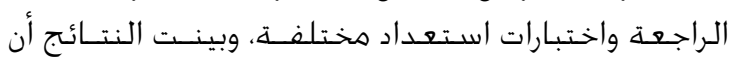

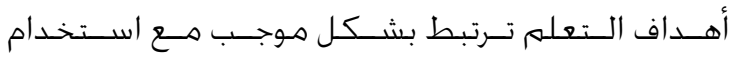
التغذية الراجعة.

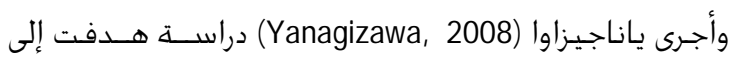

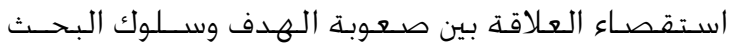

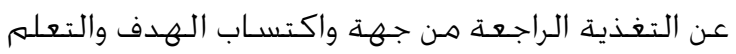

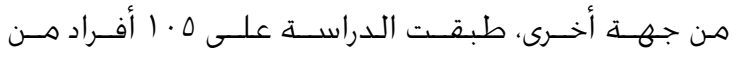

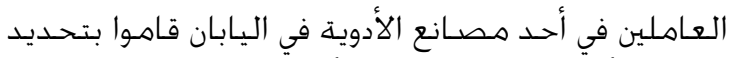

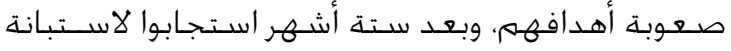

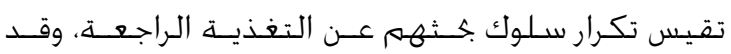

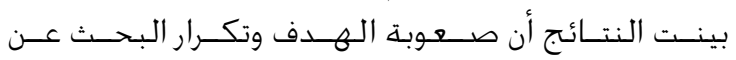

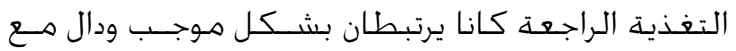

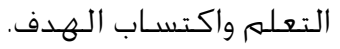

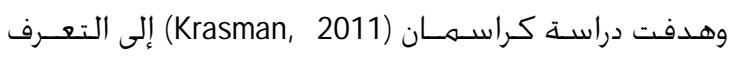

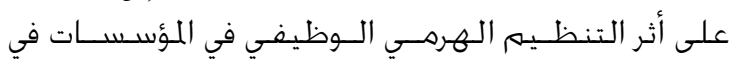

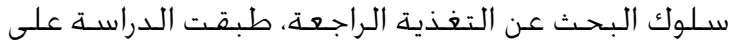

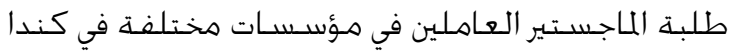

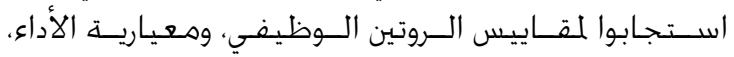

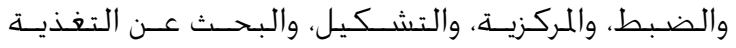

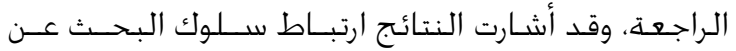

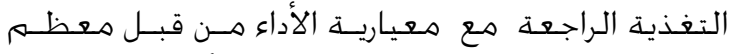

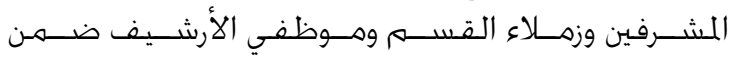
السـلم الوظيفي المعتمد.

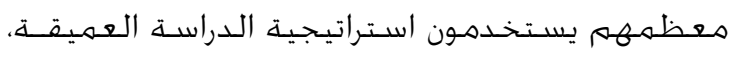

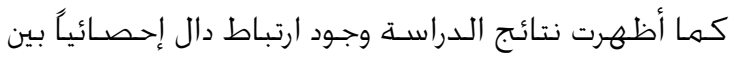

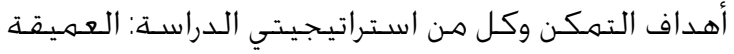

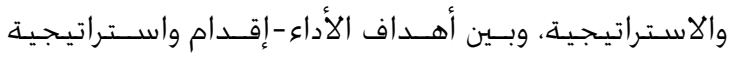

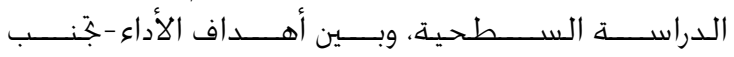
واستراتيجية الدراسـة السطحية.

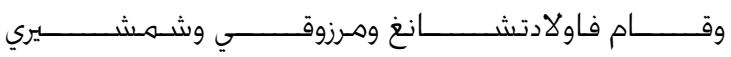
بدراســـ (Fouladchang, Morzooghi, \& Shemsheri, 2009)

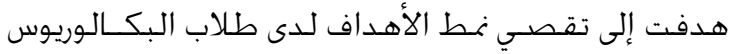

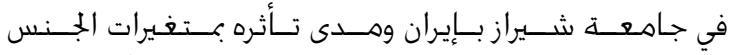

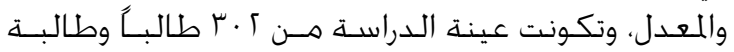

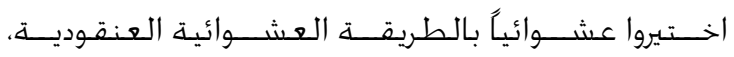

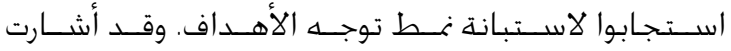

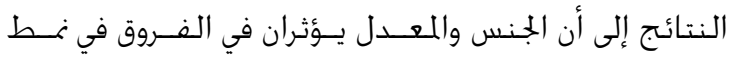

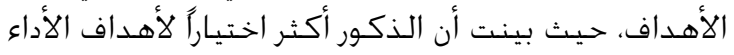

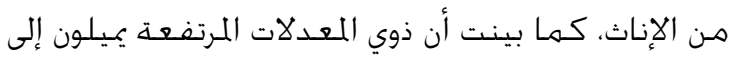

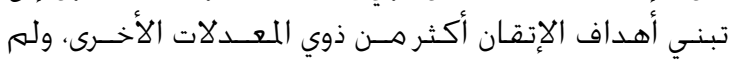
يكن هناك تفاعل بين الجنس والمعدل.

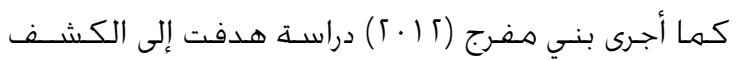

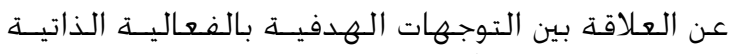

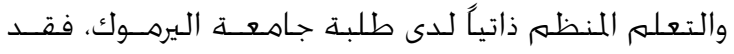

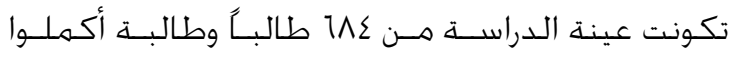

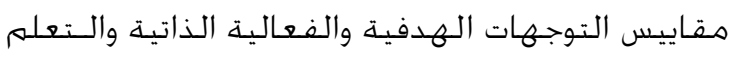

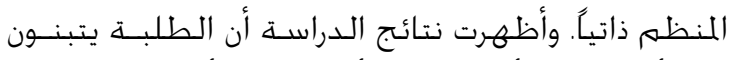

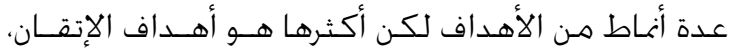

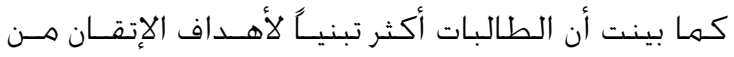

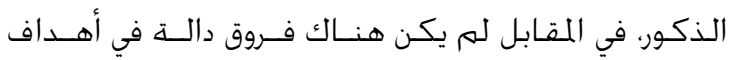
الأداء - إقدام تعزى إلى متغير الجنس.

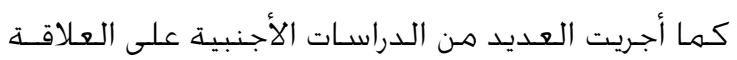

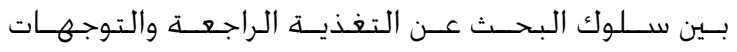

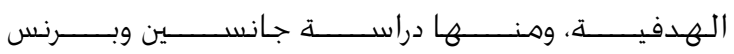
(Janssen \& Prins, 2007)

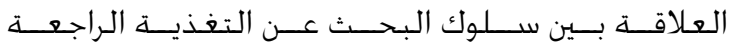

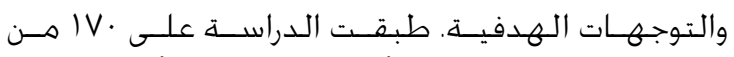

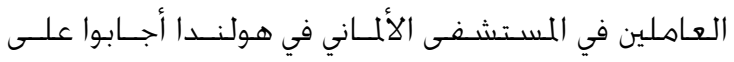

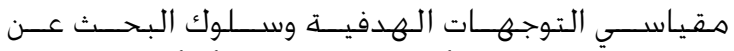

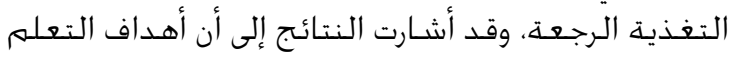

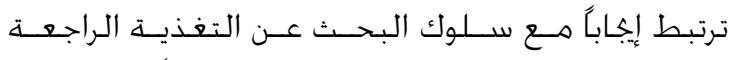

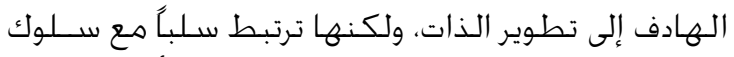

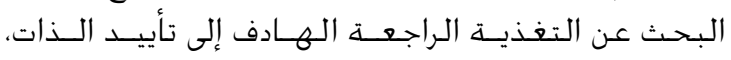

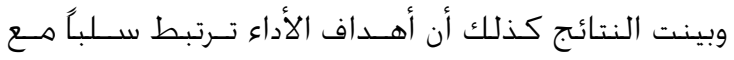

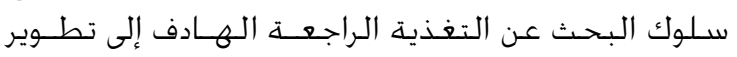

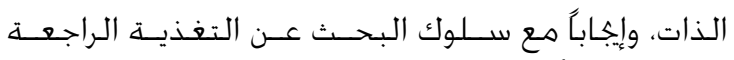

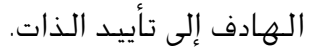


فلا توجد أية دراسـة عربية تناولت موضـوع سـلوك البحث

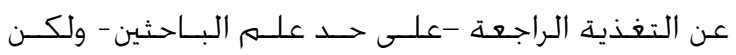

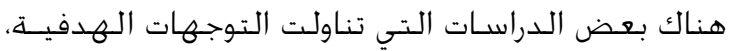

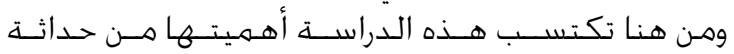

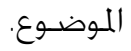

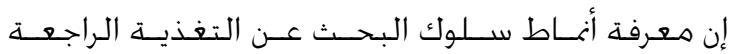

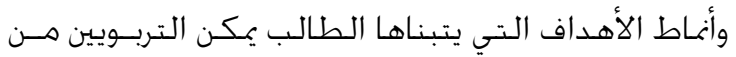

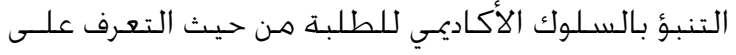

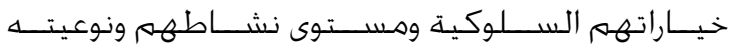
واستراتيجيات العمل لديهم (Lock \& Latham, 1990).

حدود الدراســة ومحدداتها

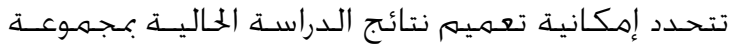

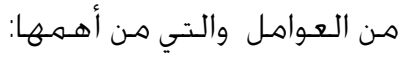

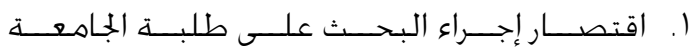

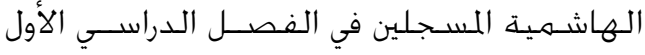

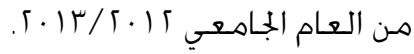

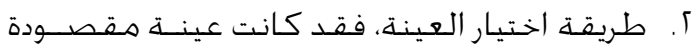

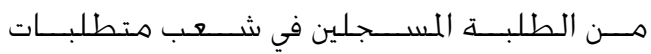

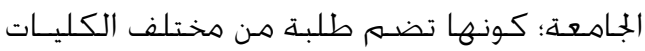

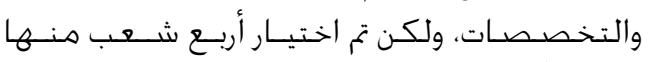
عشـوائياً.

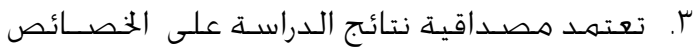

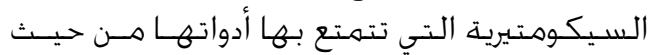
الصدق والثبات.

\section{التعريفات الإجرائية}

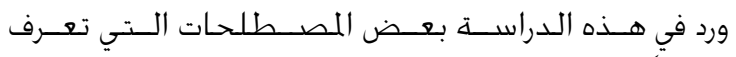

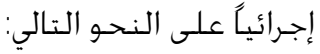

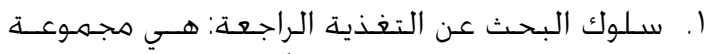

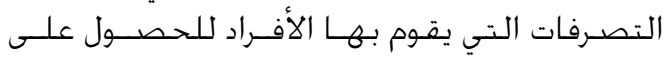

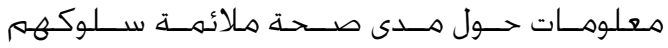
الأكاديمي، وهي تهدف إما إلى تطوير الأداء والكفاية

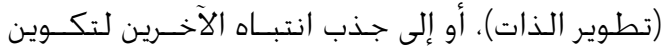

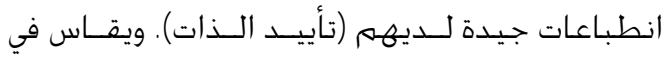

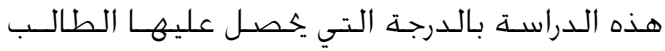

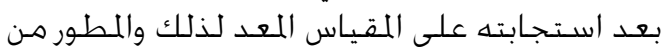
قبل (Janssen \& Prins, 2007)

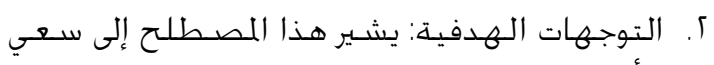

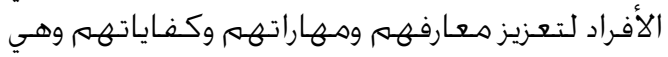
بناء نفسي ينطوي على اجزاهات وسـلوكيات تتعلق

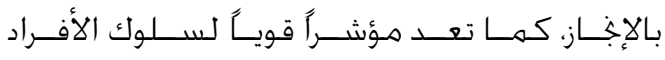

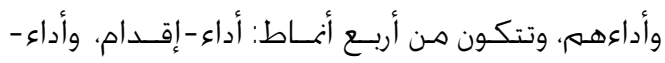

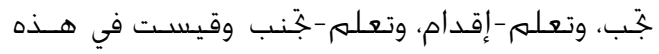

ملخص الدراسـات السـابقة وموقع الدراسة الحالية منها

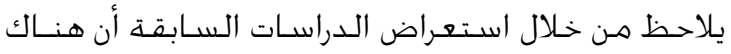

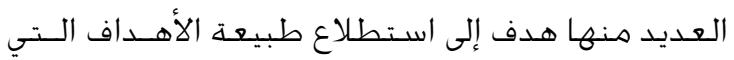

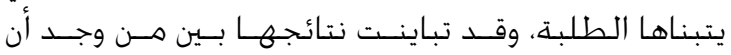

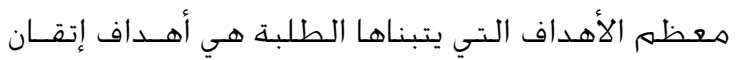

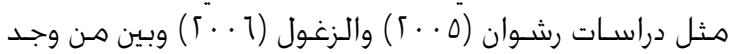

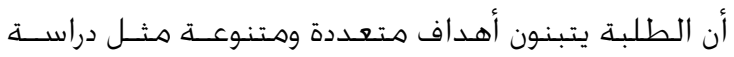

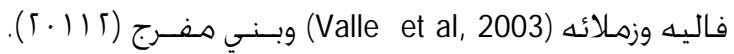

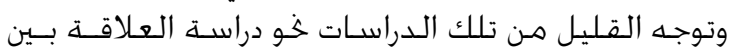

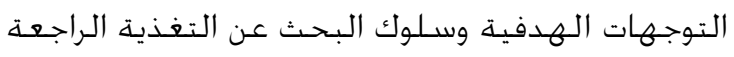

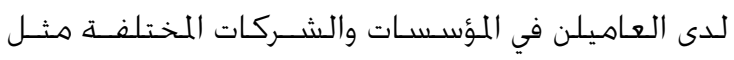

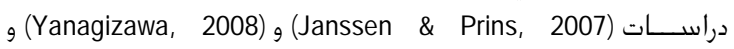

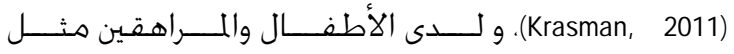

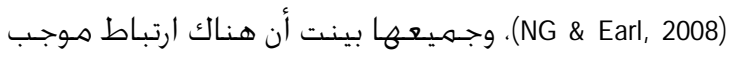

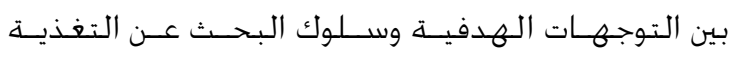

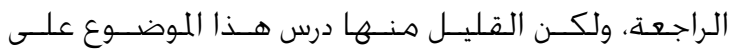

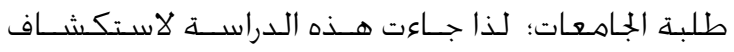

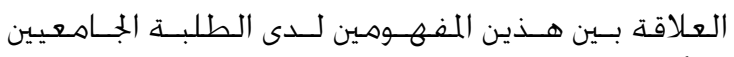

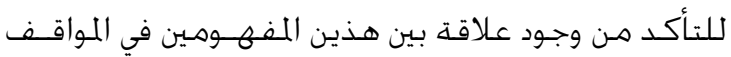

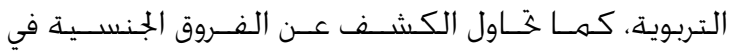

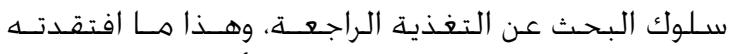
الدراسـات السـابقة، ما يضفي عليها لـفا أهمية إضـافية. مشكلة الدراسـة وأسئلتها

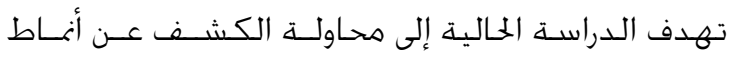

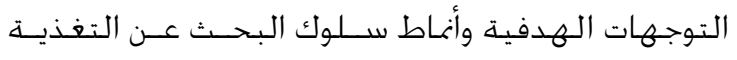
الراجعة والعلاقة بينهما لدى طلبة الجامعة الجامة الهاشـمية،

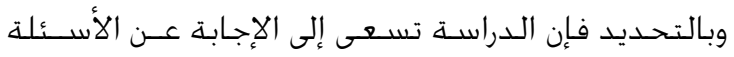

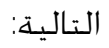

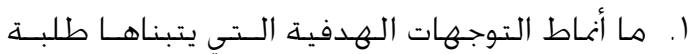

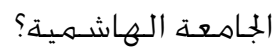

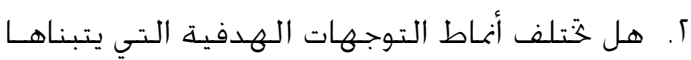

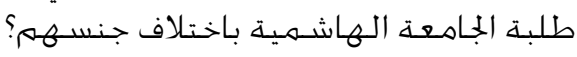

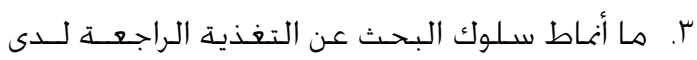

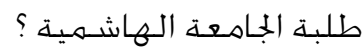
ع. هل يختلف نمط سـلوك البحتث عن التغذية الراجعة

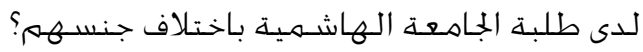

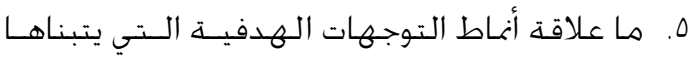

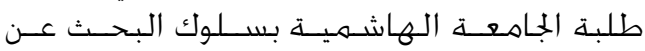

التغذية الراجعة؟ الجنامع الهاب

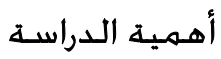

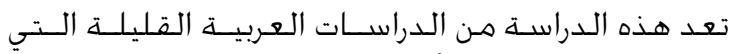

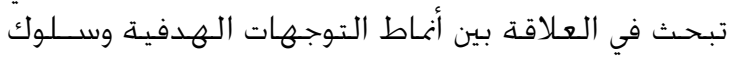

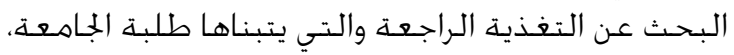


تم اسـتخـدام مقياس التوجهات الهـدفية المطور هـن قبـل

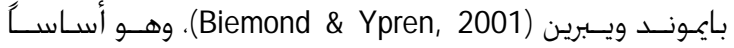

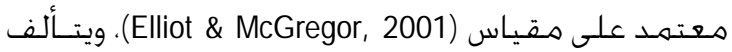

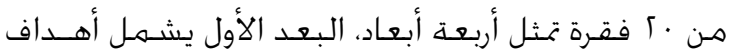

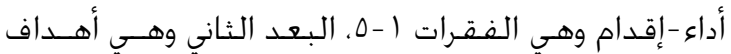

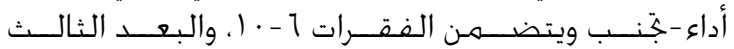

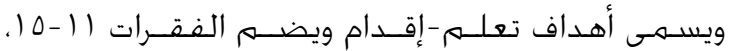

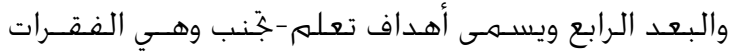

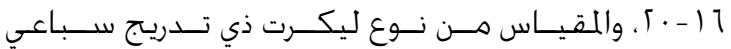

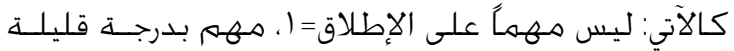

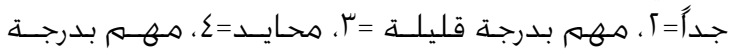

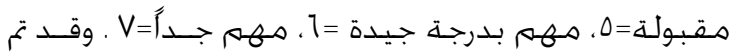

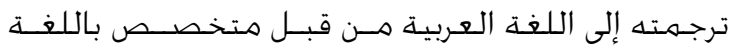

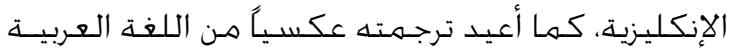

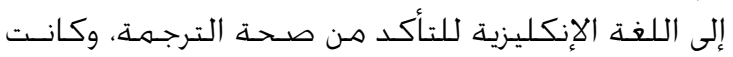

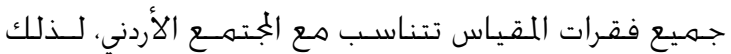

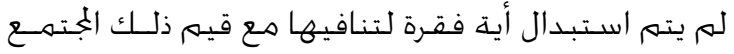

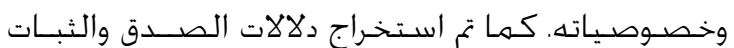
هـرة أخـرى لغايات الدراســة الخاليـة.

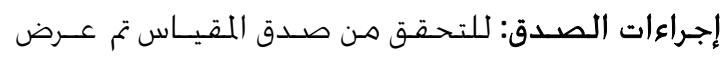

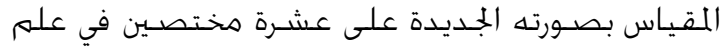

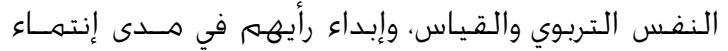

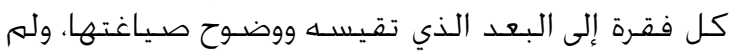

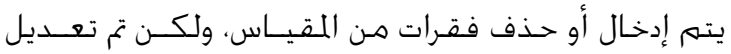

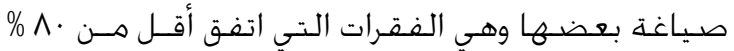

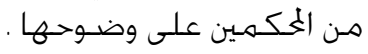
إجـراءات ثبات المقياس: تم اسـتخـراج قـيم ثبـات الاتســاق

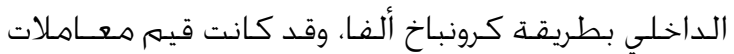

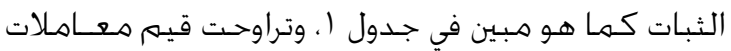

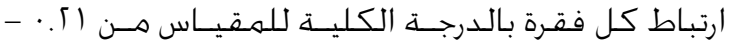
17. · وهي قيم ملاءمـة لاسـتخـدام المقياس في الــراسـة.

جدول 1

قيم معاملات ثبات الاتساق الداخلي لأبعاد

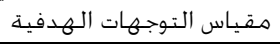

\begin{tabular}{|c|c|}
\hline قيمة معامل الثبات & البعد \\
\hline$\cdot .45$ & أداء -إقدام \\
\hline .05 & أداء -جَنب \\
\hline$\cdot .7 \mathrm{~V}$ & تعلم -إقدام \\
\hline $.9 \varepsilon$ & تعلم-جنب \\
\hline
\end{tabular}

ثانياً، مقياس سـلوك البحث عـن التغذيــــ الراجعــة تم

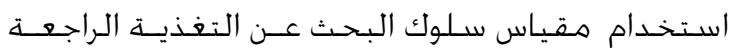

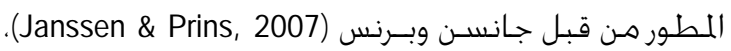

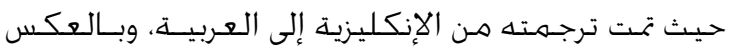

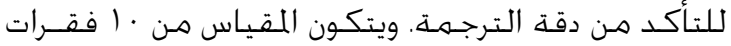

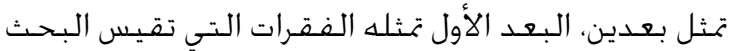

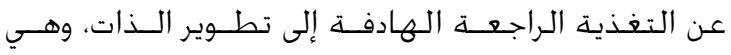

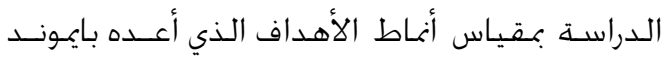

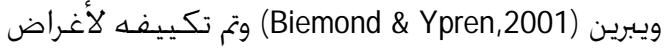
هـذه الـدراسـة.

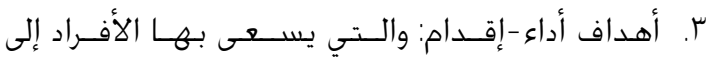

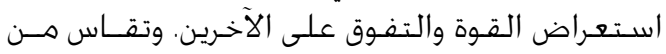

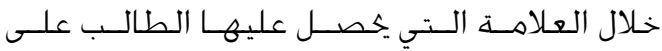
الفقـرات ا -ه هـن مقياس التوجهات الهـدفية.

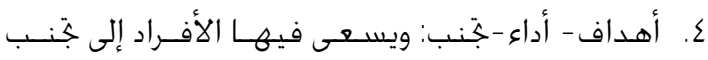

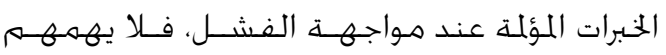

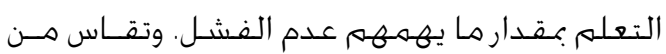

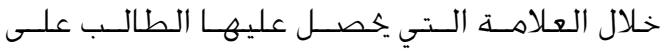

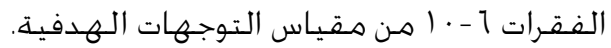

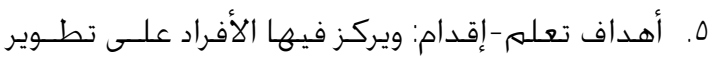

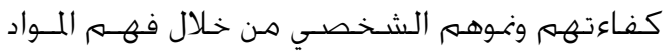

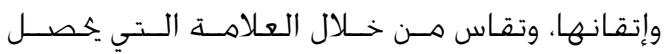

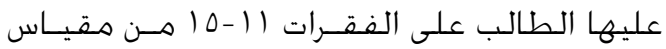
التوجهات الـهدفيتة.

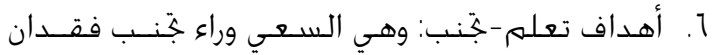

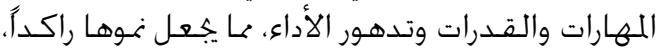

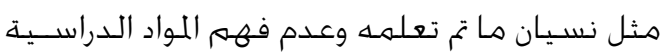

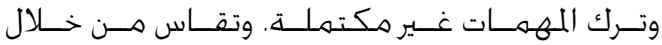

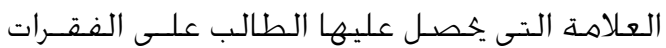

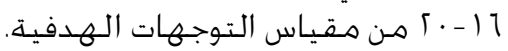

\section{الطريقة والإجراءات}

المنهج: اتبعت هذه الدراســة المنهج الوصـفي الارتباطي، للإجابة عـن أسـئلة الــراسـة.

مجتمع الدراســـ

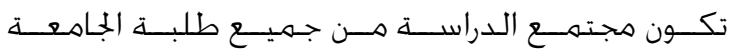

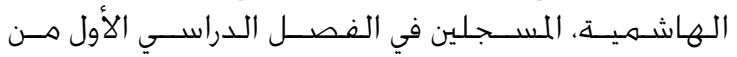

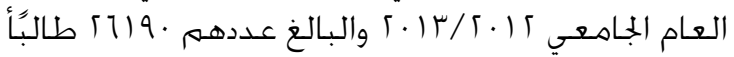

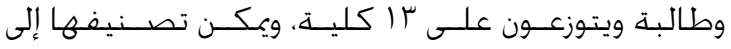
ثلاث فئات: كليات إنسـانية وكليـات علمية وكليـات تقنية.

عينة الدراسـة

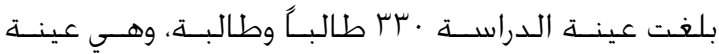

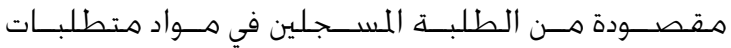

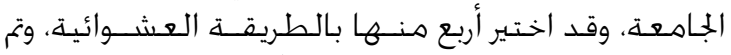

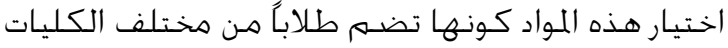

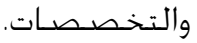

أداتا الدراسـة

أولاً، مقياس التوجهات الهدفية 
عدد منها والتي أظهر أصـحابها عدم جدية في الإجابات

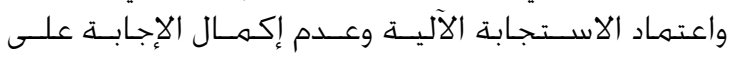

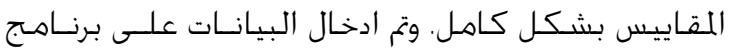

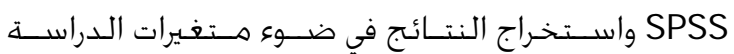

$$
\text { واهدافها. }
$$

\section{المعالجة الإحصائية}

تم خليل البيانات باستخـــام برنامـج الرزهة الإحصـائية للعلوم الاجتماعية "SPSS" حيث تم استخـراج

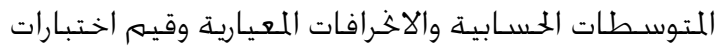

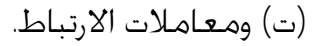

$$
\text { النتائج }
$$

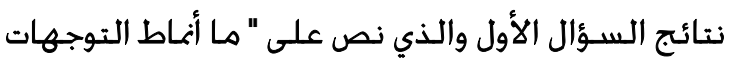

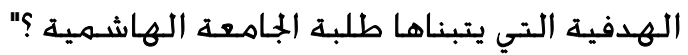

للإجابة عن السئوال الأول تم حسـاب المتوسطـات الحسابية

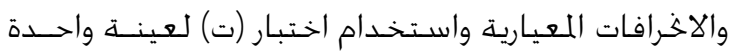

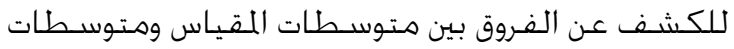

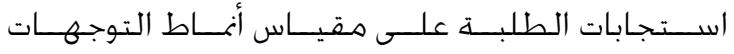
الهدفية، وجدول بّ يبين تلك القيه:

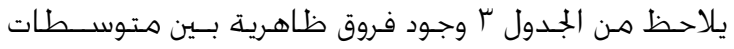

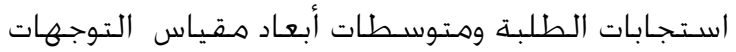

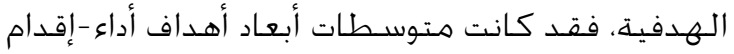

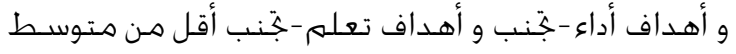

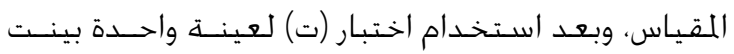

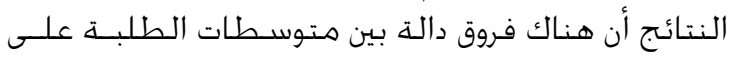

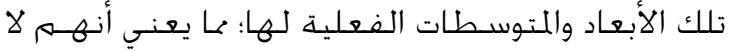

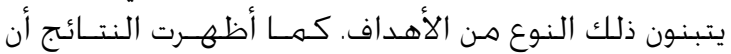

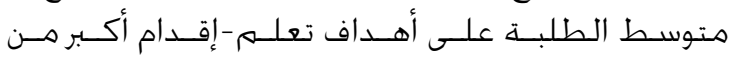

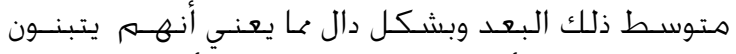

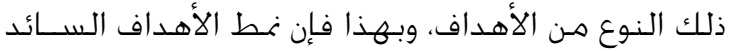
الذي يتبناه طلبة الجامعة هو أهداف تعلم -إقدام.
الفقفرات من ا - -ه. أما البعـد الثاني فتمثله الفقـرات الــي

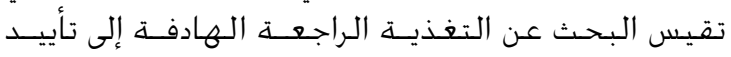

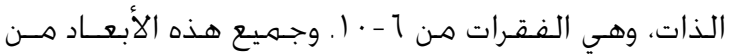

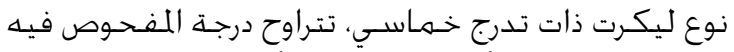

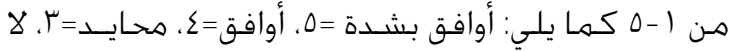

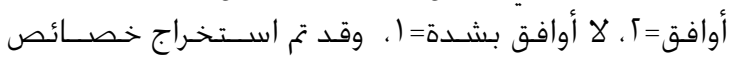
الصدق والثبات لهذا المقياس بهدف تكييفه على البيئة التهن

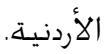

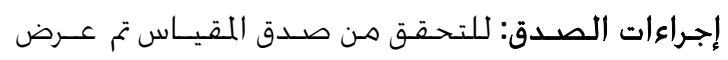

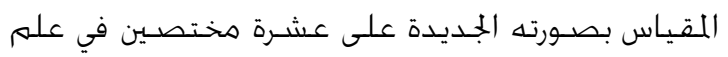

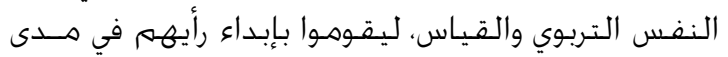

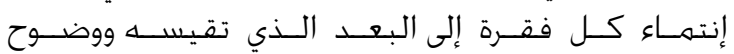

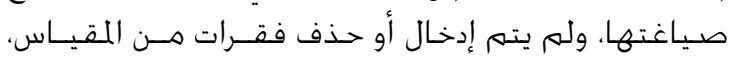

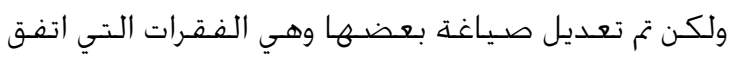

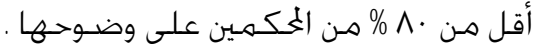

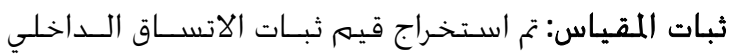

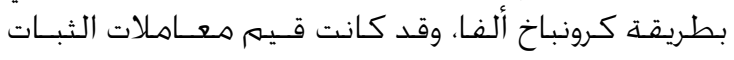

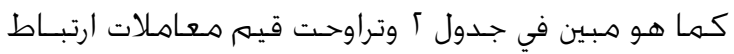

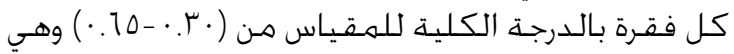
قيم ملاءمة لاستخــام المقياس في الدراسـة.

$$
\text { جـدول }
$$

\begin{tabular}{|c|c|}
\hline قيمة معـامل الثبات & البعد \\
\hline$\cdot \Lambda \Gamma$ & تطوير الذات \\
\hline.$\vee \mathrm{Q}$ & تأيـيد الذات \\
\hline
\end{tabular}

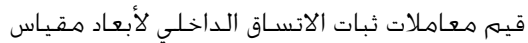

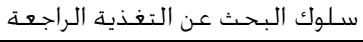

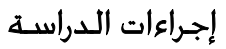

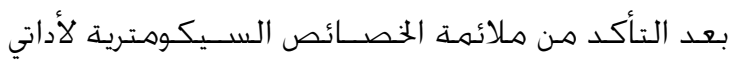

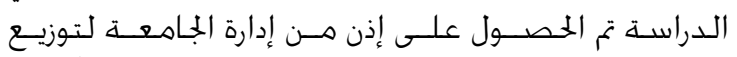

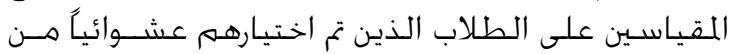

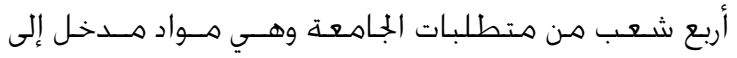

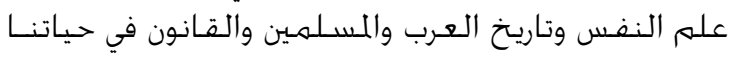

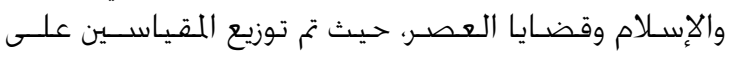

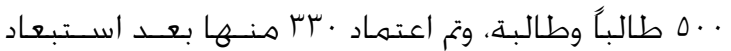

جدول ra

\begin{tabular}{|c|c|c|c|c|c|c|c|}
\hline 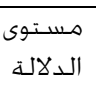 & 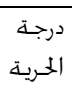 & ا ت اختبار & 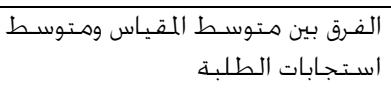 & 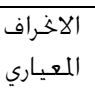 & المسابي & أبعاد المقياس & البعد \\
\hline$* \cdot, \cdots$ & $r 19$ & $\Delta V, \Delta \cdot r_{-}$ & $\wedge, \cdot \vee-$ & $\Gamma, 00$ & 11,94 & • آدرجـة & أهـداف أداء -إقـدام \\
\hline$*,, \ldots$ & riq & $1 \varepsilon, \Delta \varepsilon-$ & $9,10-$ & $\Gamma, \mathrm{V} \wedge$ & $1 \cdot, r \Delta$ & & أهداف أداء -جَنب \\
\hline$* ., \ldots$ & $r 19$ & $V \mid, r \varepsilon 7$ & 9,7 . & $\Gamma, \sum 0$ & $19,7$. & & أهداف تعلم -إقدام \\
\hline$* \cdot, \cdots$ & $r 19$ & VE, ITI- & V,I I- & I,Vo & $\mid \Gamma, \Lambda \Lambda$ & & أهداف تعلم -جنب \\
\hline
\end{tabular}

المتوسـات الحسابية والاخرافات المعيارية واختبار (ت) لعينة واحدة للكشف عن الفروق بين متوسطات مقياس التوجهات الهـدفية ومتوسطات

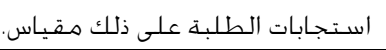


تعلم -جنَب لصـالح الذكور ما يشير إلى أنهم أكثر مسيلاً لتبني ذلك النمط من الأهداف من الإناث.

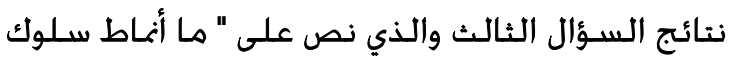

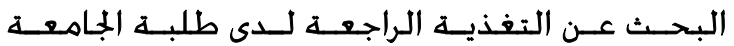

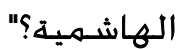

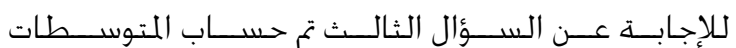

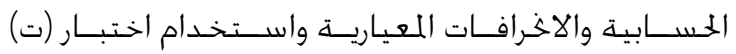

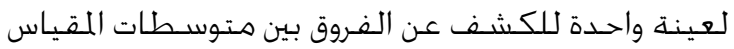

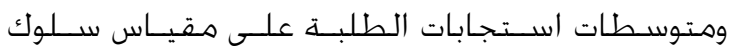
البحث عن التغذية الراجعة، وجدول ه يبين تلك القيه:

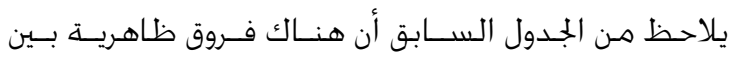

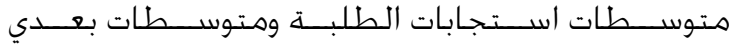

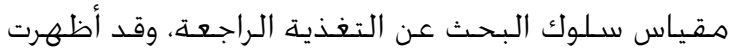

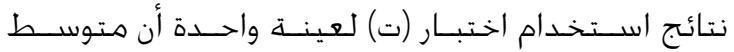

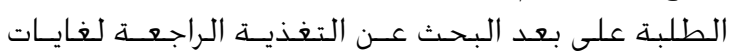

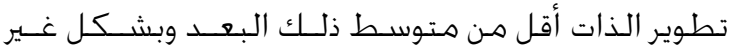

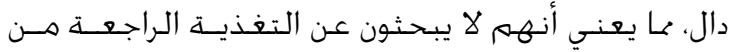

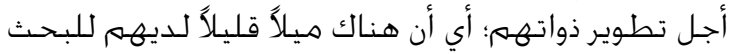

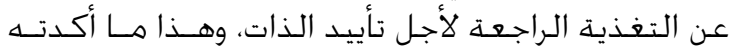

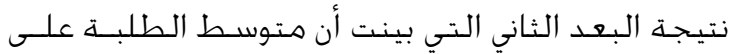

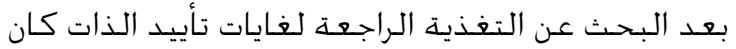

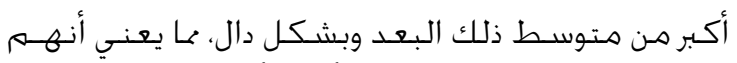

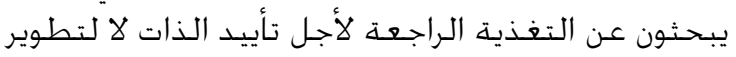

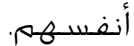

نتائج السـؤال الثاني والذي نص على" هل خَّتلف أنمـاط

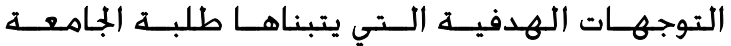
الهاشـمية باختلاف جنسهـهم؟ التهـين للإجابة عن السؤال الثاني تم حسـاب المتوسـطات الحسـابية

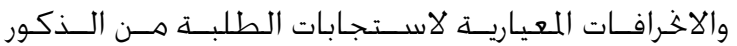

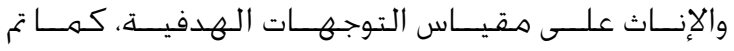

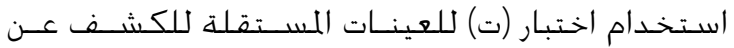

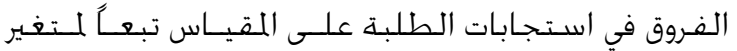
الجنس، وجدول ع يبين تلك القيه:

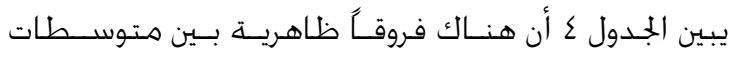

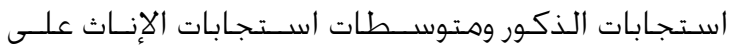

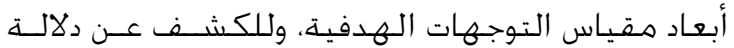

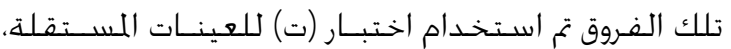

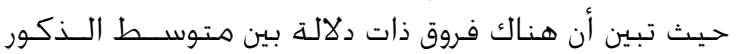

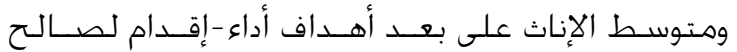

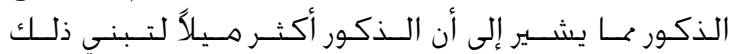

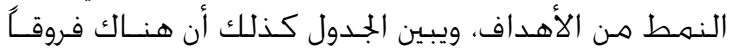

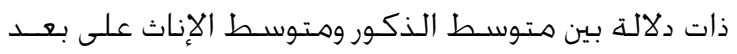

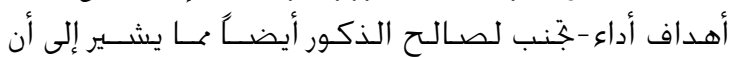

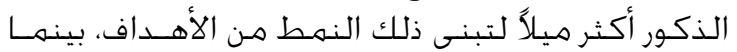

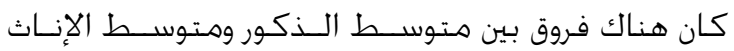

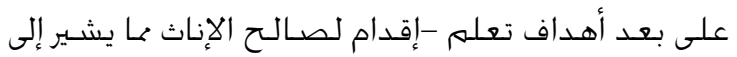

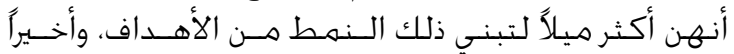

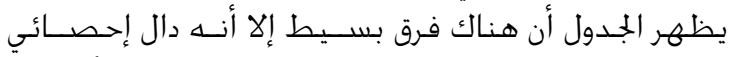

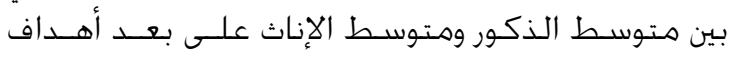

جدول

المتوسطات الحسابية والاخرافات المعيارية ونتائج اختبار (ت) للعينات المستقلة للكشف عن الفروق في استجابات الطلبة على مقياس

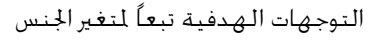

\begin{tabular}{|c|c|c|c|c|c|c|c|c|}
\hline مستوى & درجة & اختبار & الفرق بين & الاخراف & المتوسـط & العدد العد & الجذن & البعد \\
\hline الدلالة & الحرية & (ت) & المتوسـطات & المعياري & الحسـابي & & س س & \\
\hline \multirow{2}{*}{$*, \ldots$} & \multirow{2}{*}{$r \uparrow \Lambda$} & \multirow{2}{*}{$\lceil\Gamma, \Gamma \wedge\rceil$} & \multirow{2}{*}{$r, 90$} & 1,51 & $1 \%, 9 \mathrm{~V}$ & 17. & ذكور & أهداف أداء -إقدام \\
\hline & & & & 1,91 & $1 \cdot, \cdot r$ & IV. & إناث & \\
\hline \multirow{2}{*}{$*,, \cdots$} & \multirow{2}{*}{$r\ulcorner\Lambda$} & \multirow{2}{*}{$r 1, \cdot 11$} & \multirow{2}{*}{$\varepsilon, V \Gamma$} & 1,01 & I I,VA & 17. & ذكور & أهداف أداء -جَنب \\
\hline & & & & 1,50 & $\Lambda, \cdot 1$ & IV. & إناث & \\
\hline \multirow{2}{*}{$*,, \cdots$} & \multirow{2}{*}{$r\ulcorner\Lambda$} & \multirow{2}{*}{$\mu \mu, T \vee 1-$} & \multirow{2}{*}{$\varepsilon, r)-$} & $1, \cdot 9$ & $\ulcorner V, r \Lambda$ & 17. & ذكور & أهداف تعلم -إقدام \\
\hline & & & & $1, \pi \mu$ & $\mu 1,19$ & IV. & إناث & \\
\hline \multirow{2}{*}{$*,, \cdots$} & \multirow{2}{*}{ ris } & \multirow{2}{*}{$\varepsilon, 7 \cdot 9$} & \multirow{2}{*}{$\cdot, \wedge 1$} & 1,45 & $14, r^{2}$ & 17. & ذكور & أهداف تعلم -جَنب \\
\hline & & & & $1,9 \wedge$ & $1 \Gamma, \Sigma 1$ & IV. & إناث & \\
\hline
\end{tabular}

جدول 0

المتوسطات الحسابية والاخرافات العبارية و اختبار (ت) لعينة واحدة للكشف عن الفروق بين منوسطات مقياس سلوك البحث عن

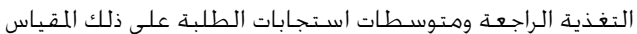

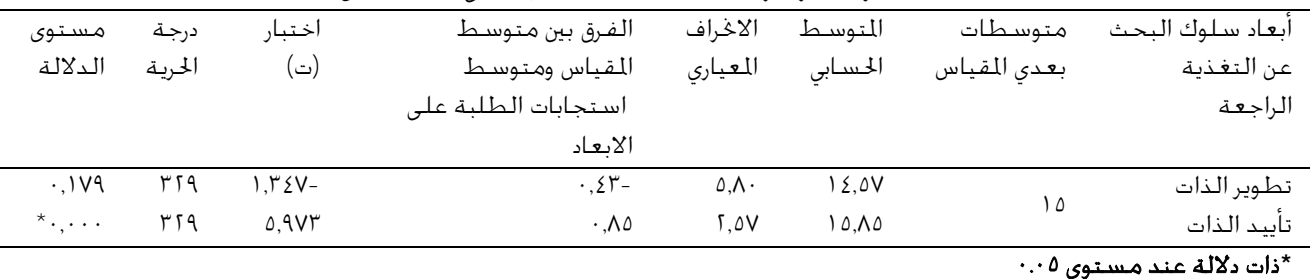




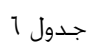

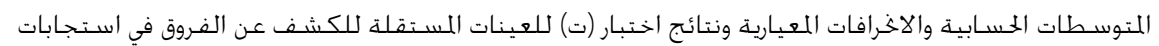

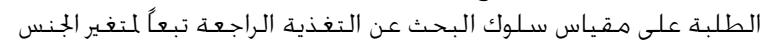

\begin{tabular}{|c|c|c|c|c|c|c|c|c|}
\hline مسـتوى & درجـة الحرية & اختبار & المتــــــــات & اللعيـاري العـراف & المستوســــي & العدد & الجــس & البعـد \\
\hline \multirow{2}{*}{$* \cdot, \cdots$} & \multirow{2}{*}{ rזA } & \multirow{2}{*}{$\sum\lceil, \Lambda \cdot\rceil-$} & \multirow{2}{*}{$1 \cdot, 1 \wedge-$} & $1,0 \Gamma$ & $4, \cdot v$ & 17. & ذكور & تطوير \\
\hline & & & & 「,V9 & $19, \mathrm{~V} 0$ & IV. & إناث & الذات \\
\hline \multirow{2}{*}{$* \cdot, \cdots$} & \multirow{2}{*}{$r\ulcorner\Lambda$} & \multirow{2}{*}{$10, \Lambda \mu V$} & \multirow{2}{*}{$r, r \wedge \Lambda$} & ז, זO & $1 \mathrm{~V}, 09$ & 17. & ذكور & تأييد الذات \\
\hline & & & & 1,09 & $|\varepsilon, \Gamma|$ & IV. & إناث & \\
\hline
\end{tabular}

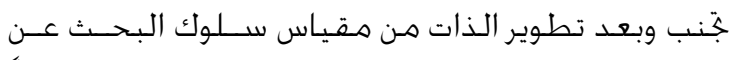

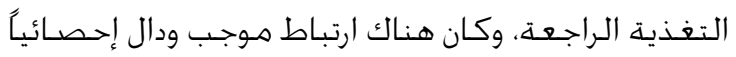

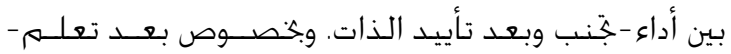

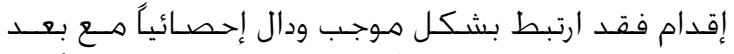

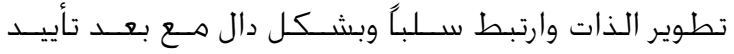

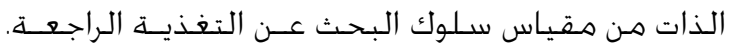

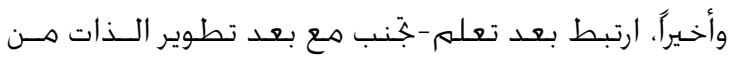

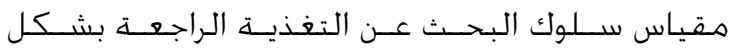

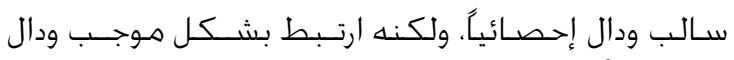
مع بعد تأييد الذات.

\section{مناقشـــ النتائج}

جدول

معاملات الارتباط بين أبعاد سـلوك البحثث عن التغذية الراجعة وأبعاد التوجهات الهدفية البحن

\begin{tabular}{|c|c|c|}
\hline تأييد الذات & تطوير الذات & المتغير \\
\hline$(* *) \cdot, \Delta \Sigma$ & $(* *) \cdot, \wedge 1-$ & أُداء -إقدام \\
\hline$(* *) \cdot, \sum 9$ & $(* *) \cdot \wedge \cdot-$ & أداء -جَنب \\
\hline$(* *) \cdot, 11-$ & $(* *) \cdot, \mathrm{V} 1$ & تعلم -إقدام \\
\hline$(*) \cdot, \mid I \Gamma$ & $(* *) \cdot, \Gamma \Delta-$ & تعلم -جنـب \\
\hline
\end{tabular}

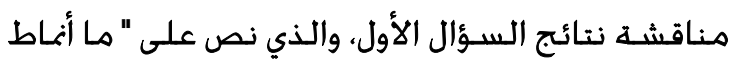

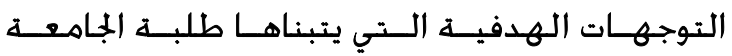

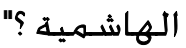

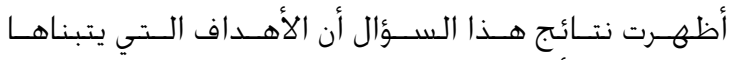

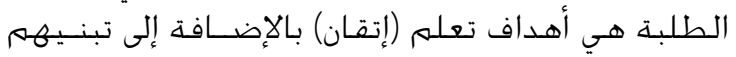

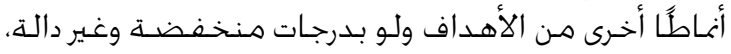

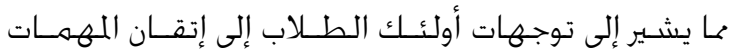

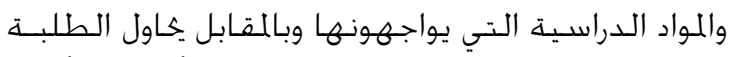

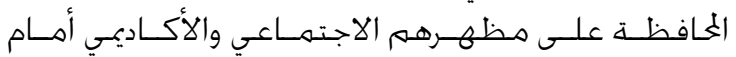

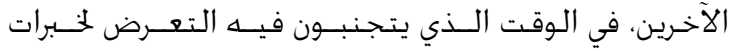

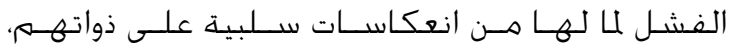

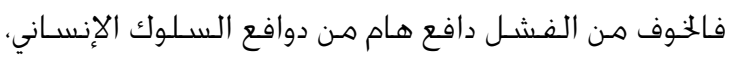

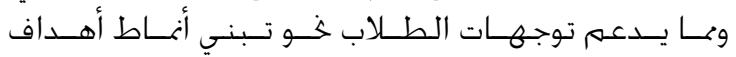

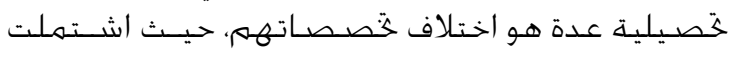

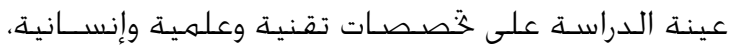

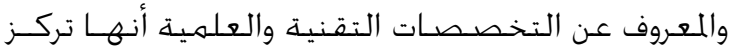

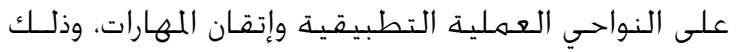

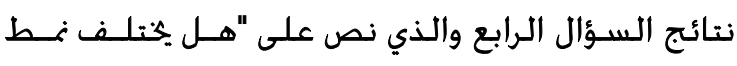

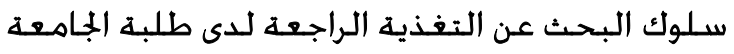

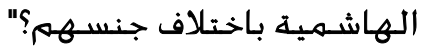

للإجابة عن السـؤال الرابع تم حسـاب المتوسـطات الحسـابيـة

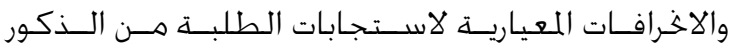

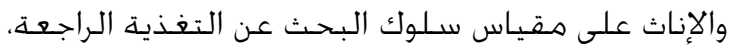

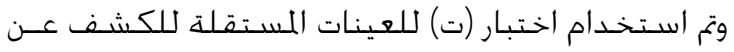

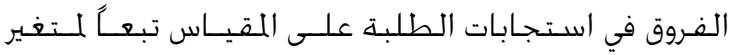
الجنس، وجـدول 1 يبين تلك القيات الطيه:

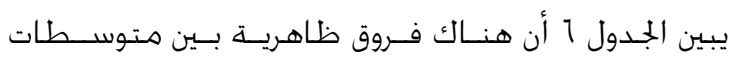

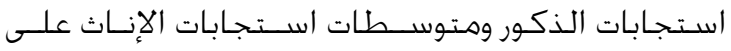

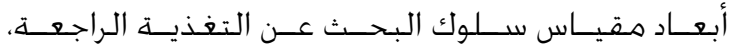

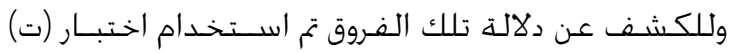

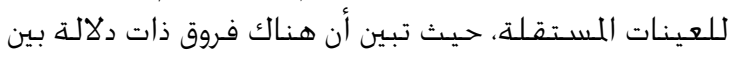

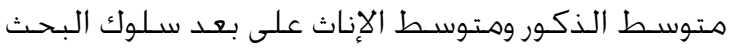

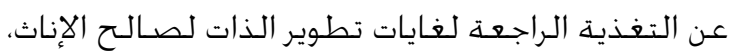

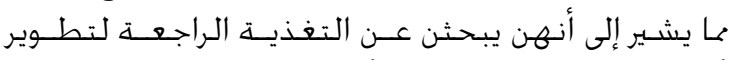

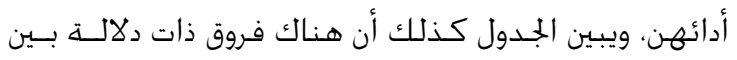

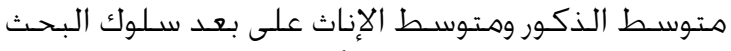

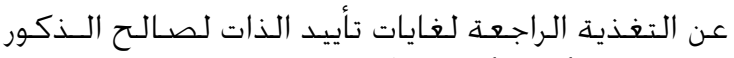

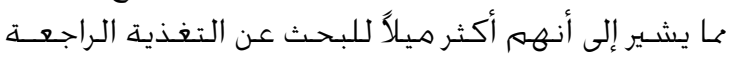

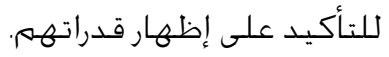

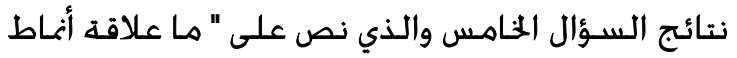

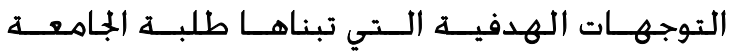

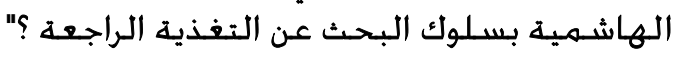

للإجابة عن هذا السـؤال تم حسـاب معـاهـل ارتباط بيرسـون

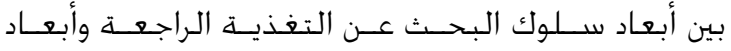

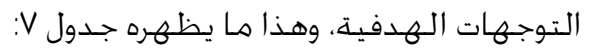
يبين جـدول V وجود ارتباط سـالب ودال إحصـائياً بين أهداف

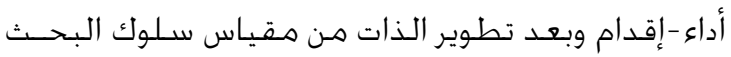

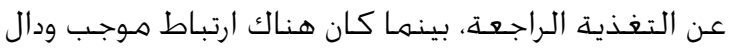

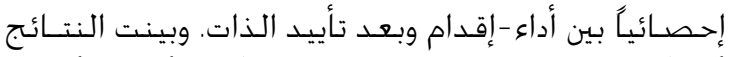

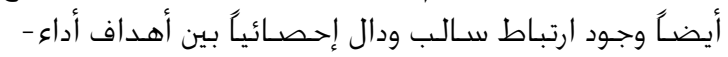




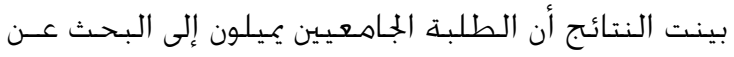

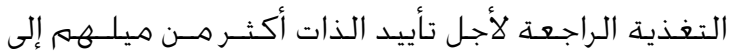

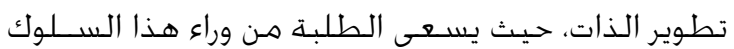

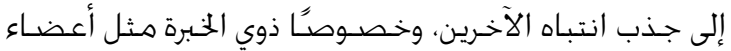

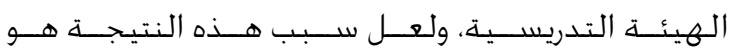

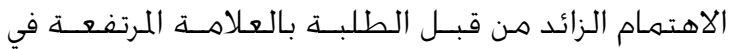

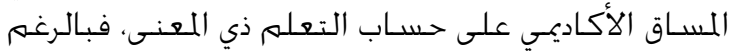

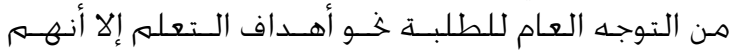

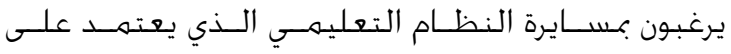

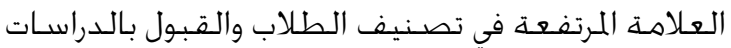
العليا وتوزيع المنح والمكافآت مـا يدفع الطلبة فئة إلى الاهتهمام

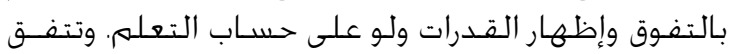

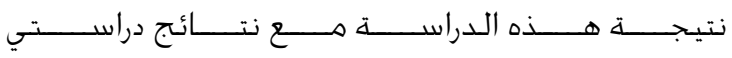
Park, Schmidt, Scheu, \& DeShon, ) (Janssen \& Prins,2007)

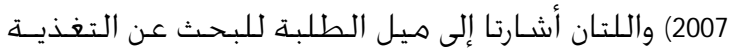
الراجعة التأييديدة.

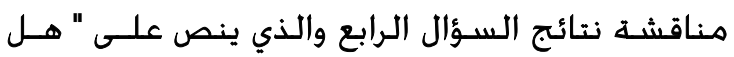

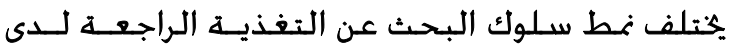

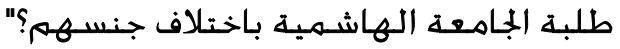

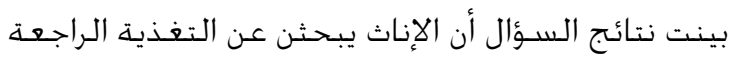

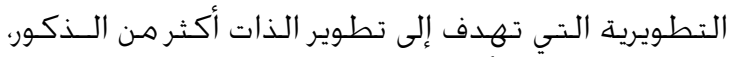

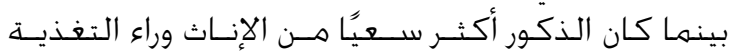

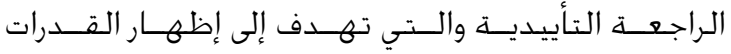

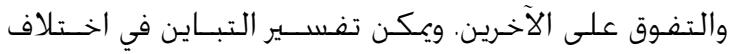

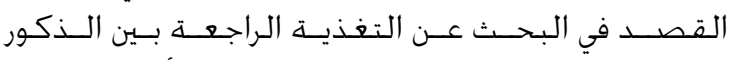

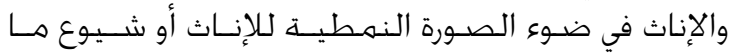

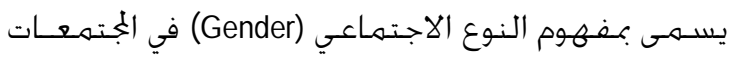

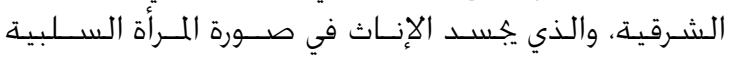

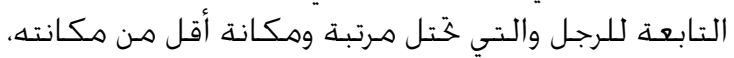

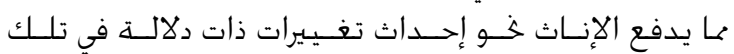

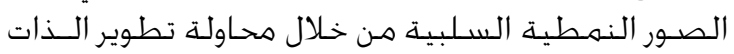

أو ما يسـهى بتمكين الذات (Self Empowerment ).

مناقشـــ نتائج السـؤال الخامس والذي ينـ علـى "مــا

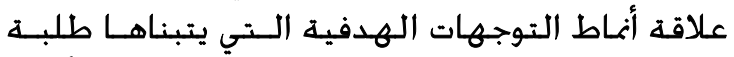

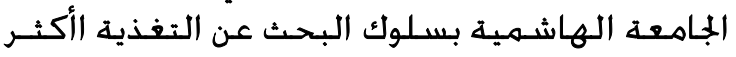

الراجعة ؟ المعه

بينت نتائج هذا السـؤال وجـود ارتباط سـالب عكسـي بـين

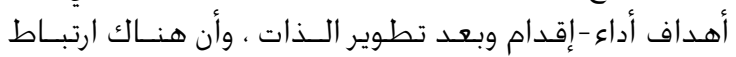

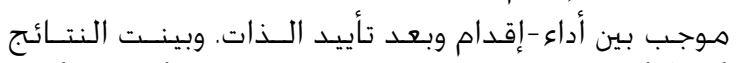

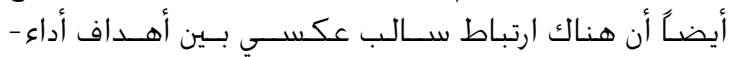

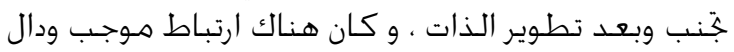

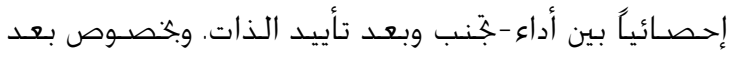

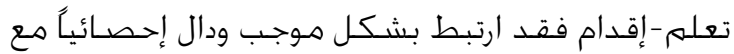

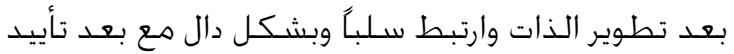

عكس ما هو متعـارف عليه في الكليات الإنســانية والـتـي

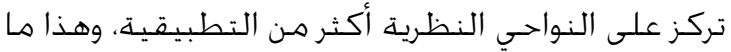

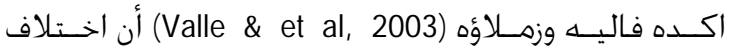

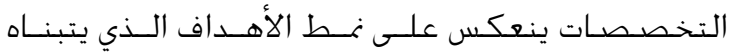

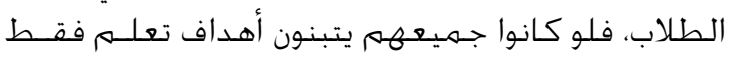

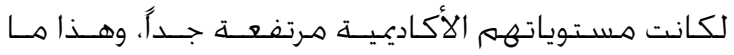

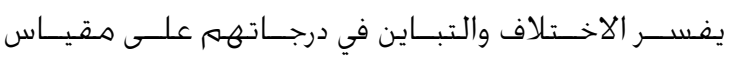

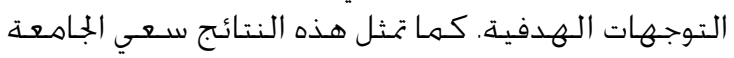

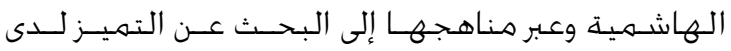

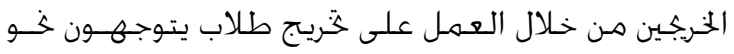

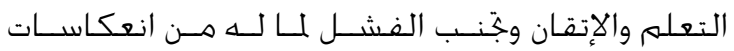

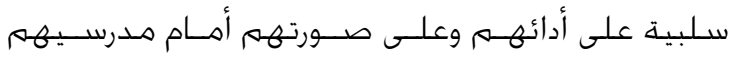

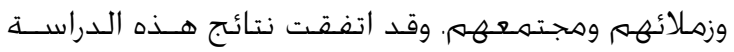

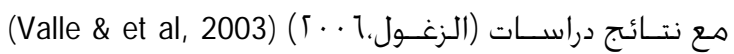

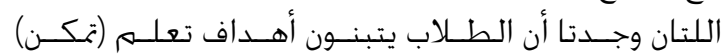
بالدرجـة الأولى.

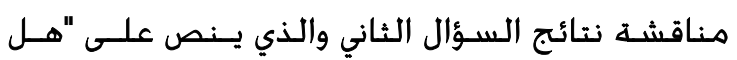

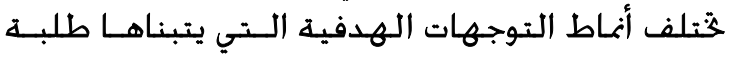

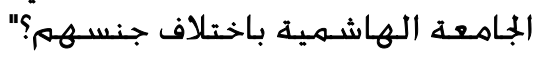

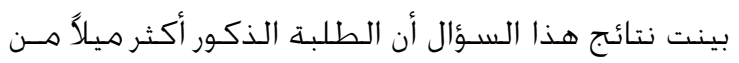

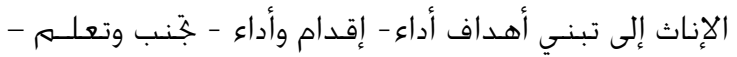

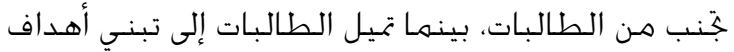

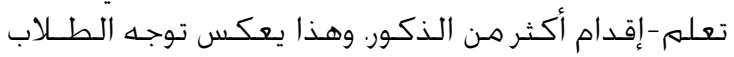

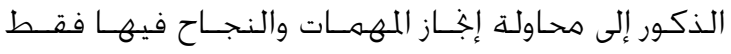

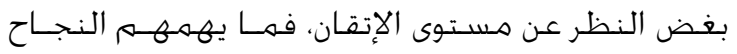

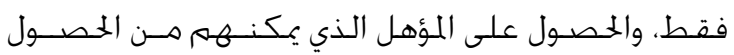

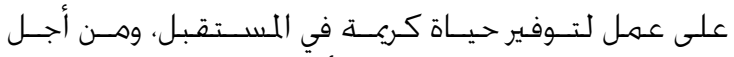

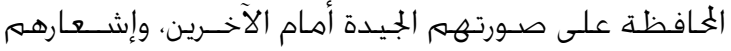

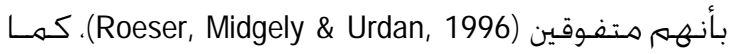

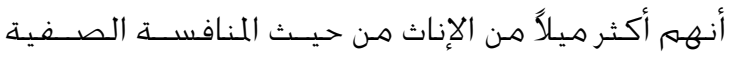

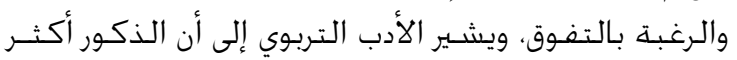

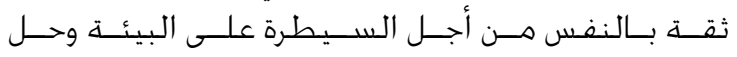

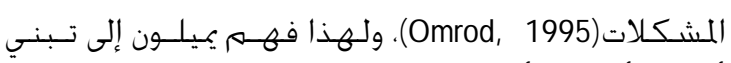

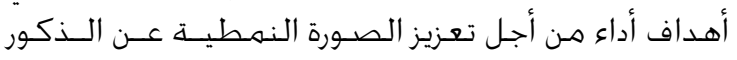

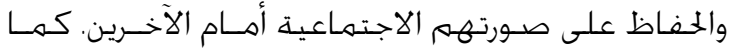

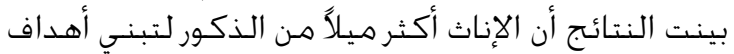

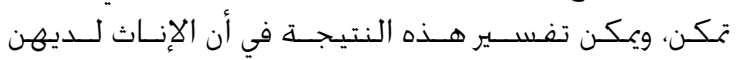

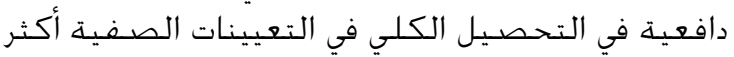

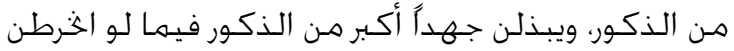

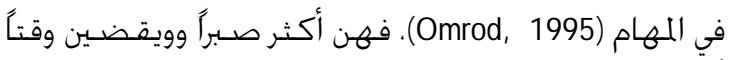

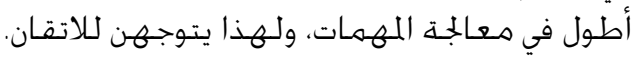

مناقشـــ نتائج السـؤال الثالث والذي ينص على " مـا أنماط

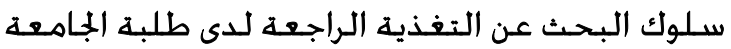

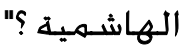




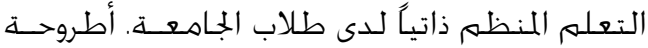

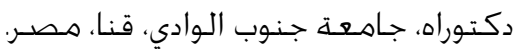

$$
\begin{aligned}
& \text { الزغـول، رافع. (1 ). أنماط الأهداف عند طلبة جامعة }
\end{aligned}
$$

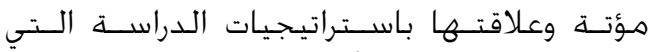

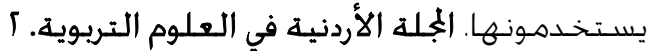

$$
\begin{aligned}
& \text {.) } 1 V-110 .(\%)
\end{aligned}
$$

غباري، ثائر. (^ · ). الدافعية: النظرية والتطبيق. عمان:

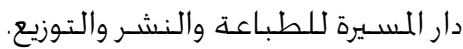

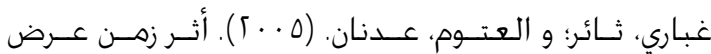

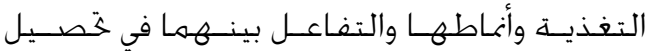

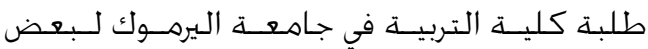

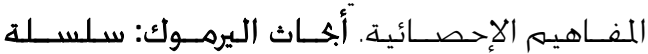

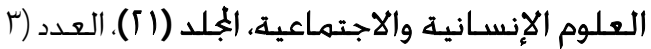

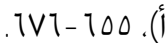

Ames, C. (1992). Classrooms: Goals, structures, and students motivation. Journal of Educational Psychology,84,261-271.

Ames, C., \& Archer, J. (1988). Achievement goals in the classroom: Students' learning strategies and motivation process. Journal of Educational Psychology, 80 (3), 260-267.

Ashford, S. J. (1986). Feedback-seeking in individual adaptation: A resource perspective. Academy of $M$ anagement Journal, 29, 465-487.

Ashford, S. J. (1989). Self-assessments in organizations. In L. L. Cummings \& B. Staw (Eds.). Research in organizational behavior (Vol. 11, pp. 133-174). Greenwich, CT: JAI Press.

Ashford, S. J., Blatt, R., \& Vande Walle, D. (2003). Reflections on the looking glass: A review of research on feedback-seeking behavior in organizations. Journal of M anagement, 29, 773-799.

Ashford, S. J., \& Cummings, L. L. (1983). Feedback as an individual resource: Personal strategies of creating information. Organizational Behavior and H uman Performance, 32, 370-398.

Bembenutty, H. (2010). A Latent Class A nalysis of Teacher Candidates' Goal Orientation, Perception of Classroom Structure, Motivation, and Selfregulation, $A$ paper presented at the annual meeting of the American Educational Research Association, Denver, $\mathrm{CO}$.

Biemond, J. M., \& Van Yperen, N. W. (2001). Generalized achievement goal orientation: Development and validation of a questionnaire. Paper presented at the WAOP-conference 2001, Tilburg, the $\mathrm{N}$ etherlands.

Dweck, C. S. (1986). Motivational processes affecting learning. A merican Psychologist, 41, 1040-1048.

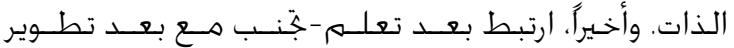

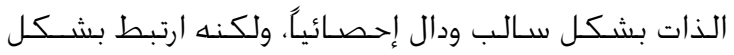

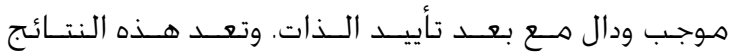

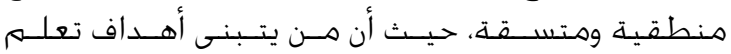

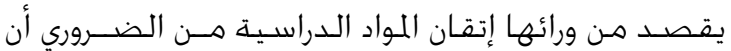

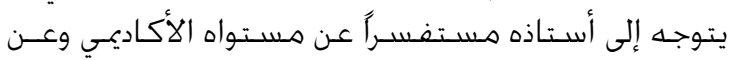

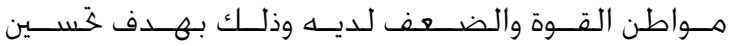

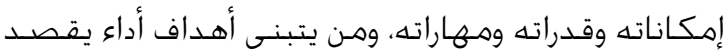

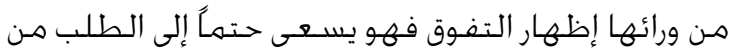

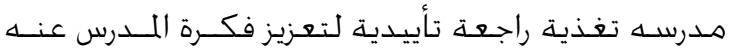

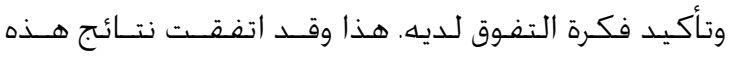

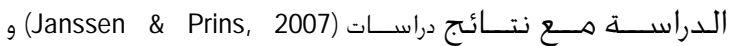
(NG and Earl, 2008) (Park, Schmidt, Scheu, \& DeShon, 2007)

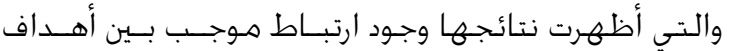

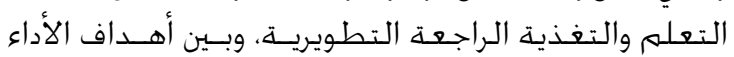
والتغذية الراجعة التأييدية.

$$
\text { التوصيات }
$$

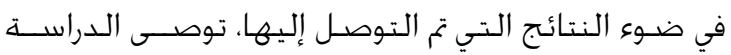
بماً يلي:

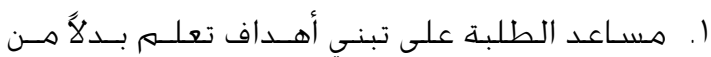

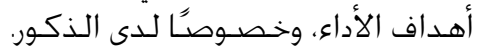

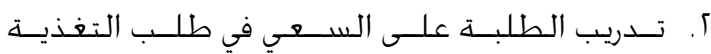

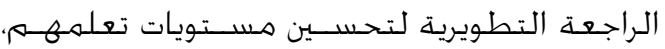

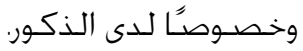

r. إجـراء المزيد هـن الـدراسـات حول مـوضـــوع التوجهــات

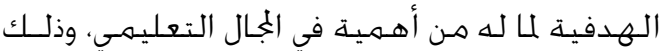

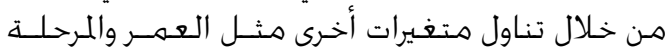

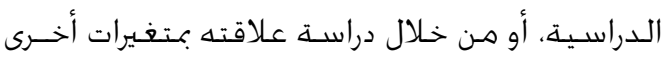

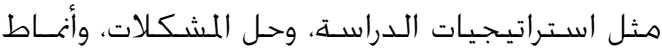

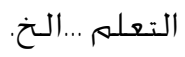

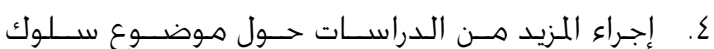

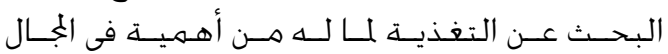

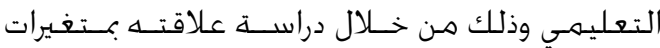
أخرى. - أخت.

\section{المراجع}

\section{References}

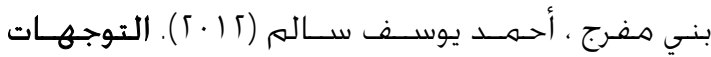

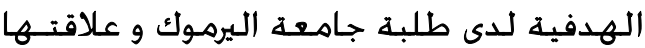

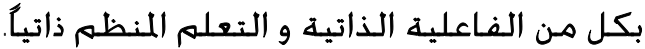

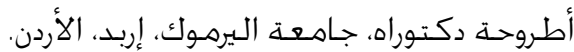

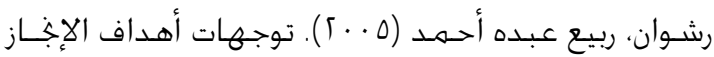

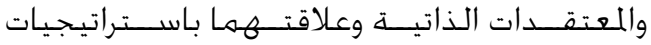


Elliot, A. J. (1999). Approach and avoidance motivation and achievement goals. Educational Psychologist, 34(3), 169-189.

Elliot, A., \& McGregor, H. (2001). A 2 × 2 achievement goal framework. Journal of Personality and Social Psychology, 80(3), 501-519.

Elliot, A. J., McGregor, H. A., \& Gable, S. (1999). Achievement goals, study strategies, and exam performance: A meditational analysis. Journal of Educational Psychology, 91, 549-563.

Elliot, A. J., \& Murayama, K. (2008). On the measurement of achievement goals: Critique, illustration, and application. Journal of Educational Psychology, 3, 613-628.

Elliott, N., Kratochwill, T., Cook; J., \& Traves, J. (2000) Educational Psychology: Effective Learning. New York: McGraw Hill

Fouladchang, M; Marzooghi, R., \& Shemshiri, B. (2009). The Effect of Gender and Grade Level Differences on Achievement Goal Orientations of Iranian Undergraduate Students. Цournal of A pplied Sciences, 9 (5), 968-972.

Hendersson, V., \& Dweck, C. S. (1990). Adolescence and achievement. In S. Feldman \& G. Elliot (Eds). At the Treshold: adolescent development. Cambridge, MA: Harvard University Press.

Janssen, O., \& Prins, J. (2007). Goal orientations and the seeking of different types of feedback information. Journal of Occupational and Organizational Psychology 80, 235-249.

Krasman, J. (2011). Taking Feedback-Seeking to the Next "Level": Organizational Structure and Feedback-Seeking Behavior. Journal of $M$ anagerial Issues 23(1), 9-30.

Kudisch, J.D., Fortunado, V.J., \& Smith, A.F.R (2006). Contextual and Individual Difference Factors Predicting Individual's Desire to Provide Upward Feedback. Group \& O rganization M anagement 31(4): 503-529.

Locke, E. A., \& Latham, G. P. (1990). A theory of goal setting and task performance. Englewood Cliffs, NJ: Prentice-Hall

Meece, J. L., Blumenfeld, P. C., \& Hoyle, R. H. (1988). Students'goal orientation and cognitive engagement in classroom activities. Journal of Educational Psychology, 80, 514-523.

Meece, J., \& Holt, K. (1993). A pattern analysis of students' achievement goals. Journal of Educational Psychology, 85, 582-590.

Nicholls, J. G. (1984). Achievement motivation: conceptions of ability, subjective experience, task choice, and performance. Psychological Review, 91(3), 328-346.

NG, J. R., \& Earl, J.K. (2008). Accuracy in selfassessment: The role of ability, feedback, selfefficacy and goal orientation . A ustralian Journal of Career Devel opment, 17(3) , 39-50.
Ormrod, J. (1995). Educational Psychology: Principles and applications. U.S.A: Printice Hall.

Park, G., Schmidt, A.M., Scheu, C., \& DeShon, R.P. (2007). A Process Model of Goal Orientation and Feedback Seeking. H uman Performance, 20(2), 119145.

Renn, R. W., \& Fedor, D. B. (2001). Development and field test of a feedback seeking, self-efficacy, and goal setting model of work performance. Journal of $M$ anagement, 27(5), 563-583.

Roeser, R. W., Midgely, C., \& Urdan, T. C. (1996). Perceptions of the school psychological environment and early adolescents' psychological and behavioral functioning in school: The mediating role of goals and belonging. Journal of Educational Psychology, 88, 408-422.

Valle, A., Cabanach, R. G., Nunez, J. C., GonzalezPienda, J., Rodriguez, S., \& Pineiro, I. (2003). Multiple goals, motivation and academic learning. British Journal of Educational Psychology, 73, 71-87.

VandeWalle, D. (2003). A goal orientation model of feedback-seeking behaviour. Human Resource $M$ anagement Review, 13, 581-604.

VandeWalle, D., \& Cummings, L. L. (1997). A test of the influence of goal orientation on the feedbackseeking process. Journal of A pplied Psychology, 82, 390-400.

VandeWalle, D., Ganesan, S., Challagalla, G. N., \& Brown, S. P. (2000). An integrated model of feedback seeking behavior: Disposition, context, and cognition. Journal of Applied Psychology, 85, 996-1003.

Was, C. (2006). Academic Achievement Goal Orientation: Taking Another Look, Electronic Journal of Research in Educational psychology, 4(3), 529-550.

Wentzel, K. R. (1989). Adolescent classroom goals, standards for performance, and academic achievement: an interactionist perspective. Journal of Educational Psychology, 81, 131-142.

Whitaker, B., J. Dahling., \& Levy, P (2007).The Development of a Feedback Environment and Role Clarity Model of Job Performance. Journal of $M$ anagement 33(4): 570- 591.

Woolfolk, A. (1995). Educational Psychology. New York: McGraw Hill.

Yanagizawa, S. (2008). Effect of goal difficulty and feedback seeking on goal attainment and learning. Japanese Psychological Research, 50 (3) 137-144.

Hseih, P., Cho, Y., \& Liu, M. (2008). Examining the interplay between middle school students achievement goals and self-efficacy in technologyenhanced learning environment. The university of Texas at san A ntonio.

Kim, Y. (2008). Effects of achievement goal orientation of dental school students on self-motivation, self- 
regulated learning strategies, and self-efficacy. Korean Journal of M edical education, 20(3), 199-208.

McWhaw, K. \& Abrami, P. (2001). Student goal orientation and interest: effects on students' use of self-regulated learning strategies. Contemporary Educational psychology.26 (3), 311-329.

Midgley, C., Anderman, E., \& Hicks, L. (1995). Differences between elementary and middle school teachers and students: A goal theory approach. Journal of Early A dolescence.15, 90-113.

Nicholls, J. (1984). Achievement motivation: Conceptions of ability, objective experience, task choice, and performance Psychological Review.91(3), 328-346.

Pajares, F. (2010).Gender and perceived self-efficacy in self-regulated learning. Theory into Practice, 41(2), 116- 125 .

Pintrich, P. (1999). The role of motivation in promoting and sustaining self-regulated learning. International Journal of Educational Research, 31(6), 459-470.

Singh, P. (n.d.). An Analysis of Metacognitive Processes Involved in Self-Regulated Learning to Transform a Rigid Learning System. Retrieved December 25, 2011.from: http:/ / www.aseesaedu.co.za/ metacog.htm.
Wolters, C. (1998). Self-regulated learning and students regulation of motivation. Journal of Educational Psychology, 90(2), 224-235.

Wolters, C., Shirley, L., \& Pintrich, P. (1996).The relation between goal orientation and students' motivational beliefs and self-regulated learning.Learning and Individual Differences, 8(3), 211-233.

Wolters, C., Yu, S., \& Pintrich, P. (2002).The relation between goal orientation and students motivational beliefs and self-regulated learning.I ndividual D ifferences, 8(3), 211-238.

Zimmerman, B. (1989).A social cognitive view of selfregulated academic learning.J ournal of Educational Psychology, 81(3), 329-339.

Zimmerman, B. (1990). Student differences in selfregulated learning: relating grade, sex, and giftedness to self-efficacy and strategy use. Journal of Educational Psychology, 82(1), 51-59.

Zimmerman, B. (1995). Self-regulation involves more than metacognitions: a social cognitive perspective. Educational Psychology, 30(4), 217-22 